\title{
HEDGE-FUND MANAGEMENT WITH LIQUIDITY CONSTRAINT
}

DOI:

10.1142/S0219024919500262

\section{Document Version}

Accepted author manuscript

Link to publication record in Manchester Research Explorer

\section{Citation for published version (APA):}

Ramirez, H. E., Duck, P., Johnson, P., \& Howell, S. (2019). HEDGE-FUND MANAGEMENT WITH LIQUIDITY CONSTRAINT. International Journal of Theoretical and Applied Finance.

https://doi.org/10.1142/S0219024919500262

\section{Published in:}

International Journal of Theoretical and Applied Finance

\section{Citing this paper}

Please note that where the full-text provided on Manchester Research Explorer is the Author Accepted Manuscript or Proof version this may differ from the final Published version. If citing, it is advised that you check and use the publisher's definitive version.

\section{General rights}

Copyright and moral rights for the publications made accessible in the Research Explorer are retained by the authors and/or other copyright owners and it is a condition of accessing publications that users recognise and abide by the legal requirements associated with these rights.

\section{Takedown policy}

If you believe that this document breaches copyright please refer to the University of Manchester's Takedown Procedures [http://man.ac.uk/04Y6Bo] or contact uml.scholarlycommunications@manchester.ac.uk providing relevant details, so we can investigate your claim.

\section{OPEN ACCESS}




\title{
Hedge Funds Management with Liquidity Constraint
}

\author{
H.E. RAMIREZ \\ Faculty of Economics, Universidad del Rosario. \\ Calle 12C No. 6-25 Bogotá, COL. \\ hugoedu.ramirez@urosario.edu.co \\ P. DUCK \\ School of Mathematics, The University of Manchester. \\ Oxford road, Manchester, M13 9PL, UK \\ duck@maths.manchester.ac.uk \\ P. V. JOHNSON \\ School of Mathematics, The University of Manchester. \\ Oxford road, Manchester, M13 9PL, UK \\ pjohnson2@maths.manchester.ac.uk \\ S. HOWELL \\ Business School, The University of Manchester. \\ Oxford road, Manchester, M13 9PL, UK \\ sydney.howell@manchester.ac.uk \\ Received (Day Month Year) \\ Revised (Day Month Year)
}

\begin{abstract}
We propose a model for a manager of a hedge fund with a liquidity constraint, where he is seeking to optimize his utility of wealth, with one and multiple period horizons. By using stochastic control techniques, we state the corresponding multi-dimensional Hamilton-Jacobi-Bellman partial differential equation and we use a robust numerical approximation to obtain its unique viscosity solution. We examine the effects of the liquidity constraint on managerial trading decisions and optimal allocation, finding that the manager behaves in a less risky manner. We also calculate the cost of being at suboptimal positions as the difference in the certainty equivalent payoff for the manager. Moreover we compare the values of a benchmark hedge fund with another one having a risky asset with a higher rate of return but less liquidity, finding that higher rate of return with a liquidity constraint does not always lead to greater return.
\end{abstract}

Keywords: Hedge fund management, stochastic control, liquidity, semi-Lagrangian, finite differences.

\section{Introduction}

Academic research into hedge funds has increased as assets under such management have risen to $\$ 3.064$ trillion in the third quarter of the 2018 according to 
BarclayHedge database ${ }^{\mathrm{a}}$. Hedge funds are virtually unregulated investments with intricate payment fees, which commonly include a manager partnership and incentive fees. According to Fung \& Hsieh (1997), hedge funds are private investment partnerships, where the managing partner is given a broad investment mandate; in addition these investments are restricted to high-worth investors. The manager of the fund is granted the authority to make trading decisions on behalf of investors or owners in the so-called managed account. Hedge-fund managers have the ability to trade on an increasing number of instruments (such as world interest rates, real estate, etc.), which could be illiquid, and in an increased number of ways such as leverage, short selling and other hedging strategies, as is also stated in Jaeger (2003). Therefore, the returns of a hedge fund will not depend solely on the initial allocation or quantity of assets but also on the trading strategy and here is where the liquidity, or lack of it, plays a fundamental role. As a consequence there is a need to study the effect of liquidity restrictions on hedge funds in some detail.

Hedge funds have been an interesting topic for research because of their distinctive form of manager compensation, their investment objectives, and their strategies, which are very different from more traditional funds, and because of the huge amounts of money these days are managed in this way. One of the key features of the manager's compensation in hedge funds is the performance-based fee or incentive fee, which is often related to a high-water mark. Typically this high-water mark is the starting fund value, and the performance fee is linked to the excess of the fund's value over the high-water mark, creating an option-like payment. Many authors have studied the consequences of this type of compensation such as Goetzmann et al. (2003), Hodder \& Jackwerth (2007), Koijen (2012), Buraschi et al. (2014) and Guasoni \& Obłój (2013) among many others. This document builds on these papers although we include into the model a key feature which has not previously been added, namely the capability of selling or/and buying a desired amount of shares within a specific time period, i.e. the illiquidity. Adding illiquidity to these models is relevant because we know that hedge-fund managers invest in different trading strategies seeking for higher returns, although in many cases these strategies are not sufficiently liquid. As a result, we found that optimal portfolio allocations and costs incurred by the manager differ from standard fully liquid cases.

A major concern about optimal portfolio allocation problems is that these often assume that assets are fully liquid and this is not always the case when investing. Therefore, we study the effects that liquidity restrictions or constraints may have on the portfolio allocation decisions of the fund's manager, in other words how restrictions affect managerial decisions. Compared with the traditional Merton (1969) portfolio optimisation problem, Hodder \& Jackwerth (2007), Guasoni \& Obłój (2013) and Fung \& Hsieh (1997) found that the optimal portfolio in hedge funds is not constant through time even with constant interest rates and volatility, and there is a need to re-balance constantly the portfolio. This paper investigates

${ }^{\mathrm{a} h t t p: / / w w w . b a r c l a y h e d g e . c o m ~}$ 
the consequences of having a non fully-liquid asset in a hedge-fund dynamic balancing portfolio. Additionally, we measure the cost of having a sub-optimal portfolio position, since a liquidity constraint does not allow the transition to an optimal position be instantaneous.

In the literature there are different ways to model liquidity, but we are interested in stock restrictions, or illiquidity in allocation, which can be seen as in Longstaff (2001), who models illiquidity as the inability to trade the desired amount of shares (between a risky and a riskless investment) within any time interval. Other types of liquidity can be found in Garleanu (2009) as the infrequent-trading feature by assuming that each agent can trade only at a subset of the time line, or as the approach of limit-order books which analyse the problem of trading at the desired price, time and amount of shares, meaning that the illiquidity is the inability to trade instantly at a given price, a given amount of shares, and that trading large amounts of shares affects the price of the asset, as in Cartea \& Jaimungal (2015), Guéant \& Pu (2017) and Çetin (2007), among many others. Longstaff's model of illiquidity may be seen as the optimal liquidation or acquisition in limit order books literature, in the sense that to eliminate the price impact the manager is only able to trade a limited amount of shares at a specific interval of time. Then, Longstaff's definition of illiquidity is appropriate for our research problem. Longstaff's model weakness is that, even with a simpler model, it has correlated terms and hence its numerical solution becomes very challenging. In this paper, we propose a novel approach to model illiquidity involving the actual amount of money in the risky and risk-less investment, which eliminates correlation variables and hence leads to a numerically tractable problem.

Our base model is structured in accordance with the papers on hedge fund management by Hodder \& Jackwerth (2007) and Guasoni \& Obłój (2013), where the fund value consists of a risky and a risk-less investment, the manager controls, or dynamically alters, the allocation between these two investments and the fund has normally distributed log returns. The manager's compensation depends on three factors: he owns a fraction of the fund (typically about 10\%), on the remaining fraction of the fund he charges an annual fee as a percentage (usually less than 5\%) and earns an incentive fee over the excess of a prescribed goal or high-water mark (typically about $20 \%$ of the excess), this incentive fee mirrors the behaviour of an European call option. In a multiple period setting, the high-water mark is adjusted depending on the manager's performance in the sense that, if at the payment time (i.e. at the end of the contract or payment period) the fund's value surpasses the high-water mark, then it must be updated to a new goal (or high-water mark) for the next period, behaviour that resembles a discrete look-back option. As stated in Guasoni \& Obłój (2013), portfolio optimisation models with finite horizons do not usually have a closed-form solution and since our model refers to portfolios with illiquid assets, which are more important to finite horizons, we do not expect a closed-form solution and therefore we use the semi-Lagrangian Crank-Nicolson numerical technique as in D'Halluin et al. (2005), to solve our problem. 
Furthermore, the fund may be closed by the manager (termed endogenous closure) or by the investor (termed exogenous closure). In the former, the manager chooses to shut down the fund, when he identifies an outside opportunity that gives him more earnings. This generates a free-boundary problem which is akin to an American option. With the exogenous closure the investor closes the fund because of poor performance. That is, if the fund falls below some established liquidation boundary (corresponding in this paper to half of the high-water mark), the investor chooses to close the fund and this type of closure is treated as a boundary.

The problem of hedge-fund management under liquidity constraints has hardly been considered in the literature previously. Adding this extra feature to the model enables us to capture the fact that hedge fund managers invest in assets with greater trading restrictions in order to gain higher returns. Aragon (2007) provides supporting results stating that greater trading restrictions are consistent with higher expected returns, which suggests that managers of hedge funds tend to invest in illiquid assets, because managers searching for higher returns would be likely to invest in some instruments that are traded less frequently (that is, more illiquid assets). By using stochastic control techniques, we are able to check the uniqueness of the viscosity solution and we solve the problem using a robust numerical scheme, i.e. The Semi-Lagrangian Crank-Nicolson. Apart from performance and returns, we explore the manager's optimal allocation, because investing in illiquid assets involves additional risk exposures (such as the inability to unwind a position before a crash). We are able to check the main differences between the value function having a fully-liquid fund and having a liquidity restriction, finding that the cost of having the liquidity constraint grows with the distance from the fully-liquid optimal portfolio position. We also consider the effects of the liquidity restriction on the manager's optimal allocation where we find that the manager behaves in a less risky manner. Additionally, we find that investing in assets with higher expected returns but less liquidity does not always lead to higher payment for the manager and that it depends on the allocation and fund's value relative to the high-water mark.

This paper is organized as follows: in section 2 we describe the model and state the problem in form of a Hamilton Jacobi Bellman (HJB) Partial Differential Equation (PDE). Section 3 presents a numerical approximation to solve the multidimensional PDE. Section 4 gives some results showing the effect of the liquidity constraint and some comparative results concerning with the effects of liquidity restrictions on the optimal portfolio and the manager's wealth. In section 5 we describe an extension of the model to allow multiple periods of time, with high-water mark resetting, which we implement as a discrete look-back option. Finally, in section 6 we give some conclusions and ideas to continue this research. 


\section{The Model}

Throughout this paper we work in a probability space $(\Omega, \mathcal{F}, \mathbb{P})$ where $\Omega$ is the event space, $\mathcal{F}$ is a $\sigma$-algebra on $\Omega$ and $\mathbb{P}$ is a probability measure, and all the random variables, stochastic processes and filtrations are defined in this space, although in the following we do not mention the probability space explicitly. Let us consider a fund comprising one risky investment and one risk-less investment; we treat the wealth of the hedge fund manager as a function of the fund's wealth and we importantly add a liquidity constraint, in the sense that we fix the maximum amount of money that can be interchanged between these two investments during a time period. Specifically, we let the manager invest in two (independent) instruments, the first is a money account which earns at a continuously compounded interest rate $r$ (the risk-less investment), and the second is the total amount of money invested in the risky asset. This risky investment relies on shares of an asset, which are usually modeled as a geometric Brownian motion with average rate of return $\mu$ and volatility $\sigma$. That is, our model distinguishes between the amount of wealth deposited on the risky investment (company shares, equity or even a portfolio of shares) and on the risk-free investment (bank account). This approach is different from the traditional way of modeling the asset price and the number of shares as two separate entities, but modeling in this way, with actual amounts of money, is convenient because it reduces one dimension and makes our problem tractable with finite differences. We call the risky instrument $R_{t}$ and the risk-less $M_{t}$.

The liquidity variable or trading strategy $\Gamma_{t}$, short for $\Gamma_{t}\left(R_{t}, M_{t}\right)$, represents the amount of money going in or out the risky investment at time $t$, so that we are restricted by the total cost of the purchase not the volume, as was assumed in Longstaff (2001). Thus at any time $t$, in a position having $R_{t}$ in the risky investment and $M_{t}$ in the riskless, the manager chooses the value of $\Gamma_{t}$ (i.e. the amount of money for buying or selling) as his strategy. The liquidity restriction is then the inability to sell more than $\left|\Gamma_{\min }\right|$ or buy more than $\Gamma_{\max }$, i.e. $\Gamma_{t} \in\left[\Gamma_{\min }, \Gamma_{\max }\right]$. This restriction on the amount of money traded at each instant of time is such that reflects the assumption that managers liquidate in order to avoid price impact on the asset, as in Cartea \& Jaimungal (2015). Although, literature in transaction costs, refer to Davis \& Norman (1990) and Cvitanić \& Karatzas (1996), states that transactions cannot be made instantly and more important costlessly, which is similar to our setting since we set limits (i.e. $\Gamma_{t} \in\left[\Gamma_{\min }, \Gamma_{\max }\right]$ ) in which we allow some transaction to be made instantly and costlessly and therefore managers do no transact outside this limits. For example, if the manager is restricted to sell (or buy) $\Gamma_{\max }=\$ 100$ in shares per period, then in a $\Delta t=0.01$ time step will only be able to trade a maximum of $\Gamma_{\max } \Delta t=\$ 1$ in shares. In this way the dynamics of the risky technology are given by

$$
d R_{t}=\mu R_{t} d t+\sigma R_{t} d B_{t}+\Gamma_{t} d t,
$$

where $B_{t}$ is a standard Brownian motion and we let $\mathcal{F}_{t}$ be the canonical filtration of $B_{t}$. The money movement between the two investments implies, for a self-financed 
portfolio, the following dynamics on the risk-less instrument

$$
d M_{t}=r M_{t} d t-\Gamma_{t} d t .
$$

The fund value is given by $X_{t}=R_{t}+M_{t}$, and the manager's wealth is given by the sum of the following:

1. The manager owns a portion $a$ of the fund, that is $a X_{t}=a\left(R_{t}+M_{t}\right)$, which is a common practice in hedge funds. In our case $a=0.1$ for an owned portion of $10 \%$. This feature is present in Hodder \& Jackwerth (2007), but not in Guasoni \& Obłój (2013).

2. The management fee is a percentage $b$ of the non-owned portion of the fund and is paid over a period of management (i.e. from 0 to $T)(1-a)\left(R_{T}+M_{T}\right) b T$, where $b=0.02$ corresponds to the $2 \%$ per period. This fee will be only paid at time $T$. If the fund is closed before $T$, the management fee will be prorated according to the actually managed time.

3. The incentive fee (or performance based fee) $c=0.2$ is paid on the excess of the fund value over some preestablished mark, the so called high-water mark $H$, so the manager earns this fee only when the fund value $X_{T}$ is greater than $H$, that is $(1-a) c\left(R_{T}+M_{T}-H\right)^{+}$. This fee will only be paid at the end of the period, or not paid at all if the fund is closed.

The manager's incentive corresponds to a call option based on the high-water mark $H$, and then the manager's terminal wealth is given by

$$
W_{T}=a X_{T}+(1-a) b T X_{T}+(1-a) c\left(X_{T}-H\right)^{+} .
$$

Remark 2.1. Although a vast part of the literature models the high-water mark as the running maximum of the wealth process, our model considers a high-water mark that is constant over some periods (payment periods should be specified in the contract). Thus the manager has a constant goal (or high-water mark) per period. First, we consider only one period dynamics implying constant high-water mark, but in section 5 , we consider a multiple period extension where the high-water mark gets reset each period (to the maximum between the values of the previous highwater mark and the fund value), meaning that, in this setting, the high-water mark resembles a discrete maximum over periods.

Investors may require closure of the fund when the fund performs poorly. The simplest approach to model investors closure is to have a lower boundary for liquidation $\Phi$, set to half of the high-water mark, i.e. $\Phi=0.5 \mathrm{H}$. In the case of an early closure of the fund because of poor performance, the manager ends up with his personal investment plus the proportional management fee, that is

$$
W_{\hat{t}}=a X_{\hat{t}}+\hat{t}(1-a) b \Phi \quad \text { for } 0 \leq \hat{t}<T
$$

here, $\hat{t}$ represents the time at which the fund hits the lower boundary, i.e. $R_{\hat{t}}+M_{\hat{t}}=$ $\Phi$. 
Furthermore, we assume the manager has some endogenous reason to close the fund, such as: leaving for a new job in a different organisation or starting a new fund. Endogenous shutdown represents an American-style option where the manager chooses when to liquidate the fund. We follow Hodder \& Jackwerth (2007) and model the manager's outside opportunities using $L$ to represent an annual external compensation rate, paid only on liquidation and not depending on the fund value. If the manager chooses to liquidate the fund at time $\tau$, he receives

$$
W_{\tau}=a X_{\tau}+(1-a) b \tau X_{\tau}+L(T-\tau) \quad \text { for } 0 \leq \tau<T .
$$

Consequently, under the incentive of winning $L(T-\tau)$, the manager is willing to liquidate if he believes that the fund is going to give an incentive less than $L(T-\tau)$. This type of closure (manager's closure) converts the problem into a optimal stopping and control problem because the manager, at each instant of time, has to revise whether is better for him to close the fund or to keep it open.

The manager seeks to maximize the expected utility of terminal wealth $W_{T}$ and has a CRRA (Constant Relative Risk Aversion) utility function with risk aversion parameter $\gamma$, namely

$$
U\left(W_{t}\right)=\frac{W_{t}^{1-\gamma}}{1-\gamma}
$$

In this problem, for each terminal fund value above $\Phi$ we calculate the utility of the obtained wealth $W_{T}$ as in (2.3), but if at any time the fund is closed, then we calculate the utility for $W_{\hat{t}}$ or $W_{\tau}$ as in (2.4) or (2.5) respectively.

Hence we seek to solve the value function

$$
J(t, R, M)=\sup _{\tau \in \mathcal{T}}\left\{\sup _{\Gamma}\left\{\mathbb{E}^{t, R, M}\left[U\left(W_{\tau}\right)\right]\right\}\right\},
$$

where $W_{\tau}$ is as in (2.3), $\mathcal{T}$ is the set of stopping times bounded by $T$ with respect to the canonical filtration $\mathcal{F}_{t}$ and $X_{T}=R_{T}+M_{T}$. The value function is subject to final condition:

$$
J(T, R, M)=U\left(W_{T}\right) .
$$

Note that $J$ depends on $R$ and $M$ rather than $W$ (manager's wealth) or $X$ (fund's value), this is because if we comprise these two variables into one ( $W$ or $X$ ) we would lose control of the liquidity restriction, because adding $R$ and $M$ cancels out $\Gamma$. Then we have a multi-dimensional value function.

We use stochastic control techniques to deduce the corresponding multidimensional HJB equation, which reflects the dynamics of the fund's value subject to a final condition at $T$, in (2.8), that is

$$
\max \left\{\mathcal{A} J+\sup _{\Gamma}\left\{(\mu R+\Gamma) \frac{\partial J}{\partial R}+(r M-\Gamma) \frac{\partial J}{\partial M}\right\}, G-J\right\}=0,
$$

where $\mathcal{A} J=\frac{\partial J}{\partial t}+\frac{1}{2} \sigma^{2} R^{2} \frac{\partial^{2} J}{\partial R^{2}}$ and $G$ is the utility of the outside opportunity, i.e. $G=G(t, R, M)=U(a(R+M)+(1-a) b t(R+M)+L(T-t))$. 
Remark 2.2. The existence and uniqueness of the solution obtained by the use of the theory of viscosity solutions and by a simple application of Crandall-Ishii and Lions' lemma to use the comparison theorem as is shown in Crandall et al. (1992), Øksendal \& Sulem (2000), Fleming \& Soner (2006) and Touzi (2013) among others. We use this theory since the value function is not smooth enough at $X=\Phi$, and then verification theorems are not applicable. Our problem is a parabolic problem for mixed optimal stopping and control, a more detailed explanation of the respective theory may be found in Touzi \& Bouchard (2011). The only issue is that we have a problem with no coercivity in the value function $J$, but it is easy to see that by using the same technique as in Crandall et al. (1992) (specifically, $J_{\epsilon}=J+\epsilon /(T-t)$ ) we derive the uniqueness condition to use Crandall-Ishii and Lions' lemma. A check of the assumptions to apply Crandall-Ishii and Lions' lemma can be found in the appendix Appendix A.

An analytical solution seems not possible because of the free boundary due to the endogenous closure condition and because we seek solutions to the optimization problem with a finite horizon, henceforth we use a numerical approach, which is explained next.

\section{Numerical approximation}

First of all, notice that we treat the risk-less $(M)$ and risky $(R)$ investments as independent entities, and under this model the HJB PDE does not involve correlated terms that would appear if we consider the wealth $X$ and constrain the number of shares to trade, as in Longstaff (2001) (In our case $\Gamma_{t}$ constrains the total cost of shares to trade and not the amount of shares to trade). Therefore, our model is numerically tractable with finite differences.

We solve the HJB PDE associated to the problem (2.7), i.e. the PDE (2.9) subject to the final condition (2.8), using a numerical approximation based on finite differences. Specifically we use the Semi-Lagrangian scheme, as in Staninforth \& Côté (1991) and D'Halluin et al. (2005), for the advection terms (corresponding to the derivatives with respect to the space variables, i.e. $R$ and $M$ ) and the CrankNicolson scheme for the diffusion terms. Then, we base our numerical scheme in Spiegelman \& Katz (2006) who implement the Semi-Lagrangian Crank-Nicolson (SLCN) scheme, and we achieve the second order accuracy in time (except on the boundary and over the region $R+M=H$ ).

First to apply the Semi-Lagrangian scheme, we use the total derivative of $J$, so that

$$
\frac{D J}{D t}=\frac{\partial J}{\partial t}+\frac{\partial J}{\partial R} \frac{d R}{d t}+\frac{\partial J}{\partial M} \frac{d M}{d t}
$$

and to match this with the advection terms of the HJB equation (2.9), we take the gradient vector coordinates to be

$$
\frac{d R}{d t}=\mu R+\Gamma, \quad \frac{d M}{d t}=r M-\Gamma,
$$


and we calculate the value of $J$ at take-off points $\left(R^{*}, M^{*}\right)$, for "each" $\Gamma$ value as

$$
\left(R^{*}, M^{*}\right)=\left(R+\alpha_{1}(R, \Gamma), M+\alpha_{2}(M, \Gamma)\right),
$$

where $\alpha_{1}$ and $\alpha_{2}$ represent the instantaneous change $\frac{d R}{d t}(R, \Gamma)$ and $\frac{d M}{d t}(M, \Gamma)$ respectively. In this way, the take-off point brings the equivalent present value of $(R, M)$ through the characteristic curves of $J$. Then, we determine the maximum value of $J$ over all the take-off points and call it $J^{*}$, that is

$$
J^{*}(t+\Delta t, R, M)=\sup _{\Gamma}\left\{J\left(t+\Delta t, R^{*}, M^{*}\right)\right\} .
$$

Once we have calculated the value of the function $J^{*}$, we proceed to solve the following differential equation (by using the Crank-Nicolson scheme)

$$
\frac{D J}{D t}+\frac{1}{2} \sigma^{2} R^{2} \frac{\partial^{2} J}{\partial R^{2}}=0 .
$$

Next we consider the numerics for the boundary conditions, and the imposed liquidation boundaries for the exogenous and endogenous shutdown.

\subsection{Boundary conditions}

We solve the problem in a square region, thus we truncate the domain by considering the region $R \in\left[0, R_{\max }\right]^{\mathrm{b}}$ and $M \in\left[M_{\min }, M_{\max }\right]$, for suitable values of $R_{\max }$, $M_{\min }$ and $M_{\max }$. For obtaining satisfactory results, we must set $M_{\max }$ to be much greater than the high-water mark (in our case twice the value of the high-water mark), and then ensure to include enough leveraged positions in the domain we set $p_{\max }$ to be the maximum leverage allowed (which in practice is about 4 or 5 times) and we must have $M_{\min } \leq\left(1-p_{\max }\right) M_{\max }$ and $R_{\max } \geq p_{\max } M_{\max }$. Now that the domain is set, we continue with the boundary conditions.

Consider the boundary where $R=0$, here there is no stochasticity on the fund meaning that all the money is in the bank account, i.e. $X_{T}=M_{T}$, then the manager's wealth depends only on the money account and we may use a discounted rate (of the bank account) as the boundary condition. To obtain a Neumann type boundary condition, we consider that for a small change in time $\Delta t$

$$
\left(e^{r \Delta t} M_{t}-H\right) \approx e^{r \Delta t}\left(M_{t}-H\right)
$$

and therefore, for the CRRA utility function (2.6), we can write the boundary condition as

$$
J(0, M, t-\Delta t)=e^{r(1-\gamma) \Delta t} J(0, M, t),
$$

\footnotetext{
${ }^{\mathrm{b}}$ In this paper we let $\mu>r>0$, and thus short selling (which would mean $R<0$ ) is not optimal because the expected future value of $R$ is greater than the actual value and therefore we use $R_{\text {min }}=0$.
} 
or as a Neumann-type condition

$$
\left.\frac{\partial J}{\partial t}\right|_{R=0}=-r(1-\gamma) J
$$

In the boundary where $R$ is sufficiently large, we assume that $\left(R_{t}+M_{t}\right)-H \approx$ $R_{t}+M_{t}$, since $R_{t} \rightarrow \infty$, hence the fund behaves as in Merton (1969), thus we reduce our model to that of Merton, i.e. the manager's wealth can be written as

$$
W_{t}=A X_{t}-D \approx A X_{t}=A\left(R_{t}+M_{t}\right)
$$

where $A=a+(1-a) b T+(1-a) c$, and $D=(1-a) c H$. Using the dynamics of the fund, and setting $p=\frac{A R_{t}}{A X_{t}} \approx \frac{A R_{t}}{W_{t}}$ and $1-p \approx \frac{A M_{t}}{W_{t}}$ we have

$$
d W_{t}^{p}=A d X_{t} \approx(r+p(\mu-r)) W_{t} d t+\sigma p W_{t} d B_{t},
$$

where again $B_{t}$ represents the standard Brownian motion and $W_{t}^{p}$ is the manager's wealth subject to $p$ (percentage) invested on $R_{t}$. Then the value function, for this simplified case, is

$$
J^{p}(W, t)=\mathbb{E}^{t}\left[U\left(W_{T}^{p}\right)\right] .
$$

Note that, for simplicity, we use $J^{p}(W, t)$ to represent the value function of the manager's wealth $W$ at time $t$ with fixed percentage $p$ invested in the risky asset $R$. Using the Feynman-Kac formula, we obtain the following PDE

$$
\frac{\partial J^{p}}{\partial t}+\frac{1}{2} \sigma^{2} p^{2} W^{2} \frac{\partial^{2} J^{p}}{\partial W^{2}}+(r+p(\mu-r)) W \frac{\partial J^{p}}{\partial W}=0,
$$

which is similar to the optimal portfolio problem described in Merton (1969), and thus has a known solution for the case of CRRA utility functions. Merton obtains closed form solutions for the optimal allocation $p^{*}$ and the value function $J$. To avoid the fully liquid effect present in Merton's solution, we use the close form solution of $J^{p}$, as a boundary condition, but instead of maximizing the portfolio $p$ we use different fixed values of $p$ depending on the actual allocation of $R$ and $M$ (i.e. $\left.p=\frac{R}{R+M}\right)$, that is

$$
J^{p}(W, t)=\exp \left\{\left(r+p(\mu-r)-\frac{1}{2} \gamma \sigma^{2} p^{2}\right)(1-\gamma)(T-t)\right\} \frac{W^{1-\gamma}}{1-\gamma} .
$$

The exponential in (3.11) can be regarded as the Merton discount rate, so we convert this to a Neumann-type condition as

$$
\frac{\partial J^{p}}{\partial t}=-\left(\left(r+p(\mu-r)-\frac{1}{2} \gamma \sigma^{2} p^{2}\right)(1-\gamma) \Delta t\right) J^{p} .
$$

Neumann boundary conditions (3.6) and (3.12) are the actual conditions to be used in the respective boundaries, note that for each boundary value in the grid, of the form $\left(R_{\max }, M\right)$, we first have to calculate $p$ in order to use (3.12). 


\subsection{Liquidation Boundaries}

We must also consider the fund's liquidation boundaries of the rectangular domain $\left[M_{\min }, M_{\max }\right] \times\left[0, R_{\max }\right]$. In this case, we set the maximum leverage to $p_{\max }=4.5$ times the money in the risky asset (which is undertaken by borrowing money from the bank, i.e. $M<0$ ). We emphasize that we only consider positive values of wealth since the exogenous boundary condition restricts the wealth to be greater than $\Phi=\frac{1}{2} H$.

The exogenous liquidation is modeled as a boundary condition, in the sense that when the fund value is less than half the high-water mark, the fund is closed with utility of manager's wealth at the closure time $W_{\hat{t}}$ as in (2.5). Note that, if a manager is close to this boundary he will try to dial down risk to zero (all $M$, no $R$ ) near the lower boundary and would never hit this boundary, but if the value of $R$ falls faster that the ability to get to a safe position (i.e. illiquidity effect) he would have to close the fund. Notice that the liquidity restriction is crucial because without it this closure would never happen and this boundary would be worthless.

The endogenous liquidation is more difficult, since at each time period we must determine the value for $J(R, M, t)$ for each value of $(R, M)$ to decide whether to close or keep the fund active, hence this problem is a free boundary between the regions to continue the fund and to close it.

If shut down is optimal, the manager closes the fund with value $X_{t}=R_{t}+M_{t}$ and the manager's terminal wealth

$$
\tilde{W}_{T}=a X_{t}+(1-a) b t X_{t}+L(T-t) \quad \text { thus } \quad J(R, M, t)=G(t, R, M)
$$

and if to continue the fund is optimal, the manager closes the fund at the final time $T$, and then

$$
J(R, M, t)=\max _{\Gamma} \mathbb{E}^{t}\left[U\left(W_{T}\right)\right]
$$

where $W_{T}$ is given by $(2.3)$. This means that $J(R, M, t)$ is described by the dynamics of the HJB in (2.9) and (2.8). Note that the max in (2.8) is a result of treating this problem as a free boundary, similar to an American option, as in Wilmott (1995) (i.e. a linear complementary problem), and solve HJB equation with a Semi-Lagrangian Crank-Nicolson PSOR scheme.

\section{Results}

In this section, we describe and explain results for the problem of the manager's optimal expected utility of wealth, using a $C R R A$ utility as the function representing the preferences of the manager. To be able to compare our results with those in Hodder \& Jackwerth (2007), we use their same set of parameters, shown in table 4. In this table, we add the constant $\mathfrak{p}=\frac{\mu-r}{\sigma^{2} \gamma}$ to resemble the optimal allocation for the simplified problem (i.e. Merton's problem), and we perform a segment search for the maximum argument of $\Gamma$ in the appropriate range. For the purposes of this 
Table 1. Model parameters, as in Hodder

\& Jackwerth (2007)

\begin{tabular}{cc|cc}
$T_{\max }$ & 1.0 & $\Delta t$ & 0.02 \\
$\Gamma_{\max }$ & 0.3 & & \\
$R_{\text {initial }}$ & 0.0 & $R_{\text {final }}$ & 8 \\
$\Delta R$ & 0.01 & & \\
$M_{\text {initial }}$ & -6 & $M_{\text {final }}$ & 2 \\
$\Delta M$ & 0.01 & & \\
$\mu$ & 0.0778 & $\sigma$ & 0.05 \\
$r$ & 0.0578 & $\gamma$ & 4 \\
$H$ & 1.0 & $L$ & 0.018 \\
$a$ & 0.1 & $b$ & 0.02 \\
$c$ & 0.2 & $\mathfrak{p}$ & 2
\end{tabular}

paper we have symmetric liquidity constraint, i.e. of the form $-\Gamma_{\max }=\Gamma_{\min }$, although our model supports liquidity restrictions where $-\Gamma_{\max } \neq \Gamma_{\min }$.

Before considering our results we note that Hodder \& Jackwerth (2007) solve the manager's optimal portfolio allocation $p^{*}(t, X)$ (i.e. the percentage invested in the risky investment that gives the manager the maximum utility of wealth for a given fund value at a given time) having endogenous and exogenous shut down constraints. In this way the natural connection between our model's optimal trading decision $\Gamma_{t}$ with the optimal portfolio of Hodder \& Jackwerth (2007) is performed by searching the maximum utility of wealth over the diagonal $R=X-M$ for each fixed fund value $X$, that is

$$
p^{*}(t, X)=\left\{\frac{R}{R+M}:(t, R, M)=\underset{R+M=X}{\arg \max }\{J(t, R, M)\}\right\} .
$$

Figure 1 shows the optimal trading strategy $\Gamma^{*}$ for a manager with liquidity constraint $\Gamma_{\max }=0.3$, at two different times. First notice that the plotted line is to the optimal position for the manager, which corresponds to $\Gamma^{*}=0$, because his best trading decision consists on staying at this position (not selling or buying). The dashed line encloses the endogenous closure region. The resulting strategy is a bang-bang strategy, which means to one side of the curve $\Gamma^{*}=0$ sell as much as you can to get to the optimal and on the other side buy. The $\Gamma^{*}=0$ curve may be referenced by the high-water mark $R+M=H=1$ (gray dotted) line and by Merton's optimal strategy $R=-\mathfrak{p} M$, because strategies with fund values above the high-water mark (above-right) behave similarly to the optimal portfolio allocation described by Merton but with a lower leverage (i.e. $p^{*} \approx 1.55<\mathfrak{p}$ ). The figure may be interpreted in the following way, if the values correspond to a position with portfolio greater than $p^{*}$ then the decision is to sell risky assets $\Gamma^{*}=-\Gamma_{\max }$ (the top left of the graphs), and if the position has a portfolio lower than $p^{*}$ then buy $\Gamma^{*}=\Gamma_{\max }$ (bottom right of each graph). For values below the high-water mark diagonal $R+M=H$ (below-left), the strategy varies in time as we can see in the graphs at times $t=0.8$ and $t=0$. The difference between these graphs is that in the optimal curve (corresponding to $\Gamma^{*}=0$ or $p^{*}$ ) the spike across the high-water 
mark diagonal becomes less pronounced and thicker, which means that at initial time the manager, who is close to the high-water mark, behaves in a way that is less risky. Thus in the presence of the incentive, the manager shifts from a risk-seeking behaviour (before obtaining the incentive $X_{t}<H$ ) to a risk-averse behaviour (after obtaining the incentive $X_{t}>H$ ), which agrees with the findings in Guasoni \& Obłój (2013).

From calculations of equation (4.1) and the comparison to the optimal trading decisions, we can see that the optimal portfolio $p^{*}$ allocations coincide with the optimal trading decision of $\Gamma^{*}=0$. Moreover, in this setting our results agree with those found in Hodder \& Jackwerth (2007).

Figure 1 also shows that trading decisions are dynamic and depend in time and allocation of the wealth, contrary to Merton's optimal portfolio problem where the optimal allocation does not change through time and hence the decisions, at any time, are to stay at Merton's optimal allocation $\mathfrak{p}$. This dynamic strategy differentiates the kinds of problems faced in hedge funds and mutual funds and gives more importance to the liquidity restriction, because in hedge funds the manager has to trade to mantain the optimal position, which agrees with the empirical data analysis shown in Fung \& Hsieh (1997).

\subsection{Liquidity effects on the optimal portfolio}

Next, we compare our results with those in the fully liquid case. To do so, we consider the model following Hodder \& Jackwerth (2007) and solve it via stochastic control to obtain the following HJB PDE

$$
\max \left\{-\frac{\partial J_{L}}{\partial t}-\max _{p}\left\{\frac{1}{2} p^{2} \sigma^{2} X^{2} \frac{\partial^{2} J_{L}}{\partial X^{2}}+(r+p(\mu-r)) X \frac{\partial J_{L}}{\partial X}\right\}, G-J_{L}\right\}=0,
$$

where we use the notation $J_{L}$ for the value function, which is now fully liquid and depends on the total fund $X$ and the portfolio $p$. Solving this equation subject to the same closure conditions and payment fees as before involves some numerical difficulties such as using the upwind differencing, this is discussed in Ramirez et al. (2018). Although essentially, the main technique used to solve this PDE is the Crank-Nicolson PSOR.

In figure 2, we show the graph for the optimal allocation of the manager at time $t=0$, for a hedge fund with and without the liquidity constraint. We can observe that adding the liquidity constraint affects the manager's optimal allocation in the sense that near the endogenous closure (at $X \approx 0.6$ ) a manager having a liquidity restriction takes less risks in optimal positions, i.e. $p^{*}<p_{L}^{*}$ (here $p_{L}^{*}$ stands for the optimal liquid portfolio). This is due to the fact that in the case of adverse movements of the assets, the contraint in liquidity does not let the manager react fast enough to change his position. We also compare the optimal $p^{*}$ at different times $(t>0)$ and check that the differences, between the optimal portfolios with and without liquidity constraint, decrease with time. This result is expected because the 

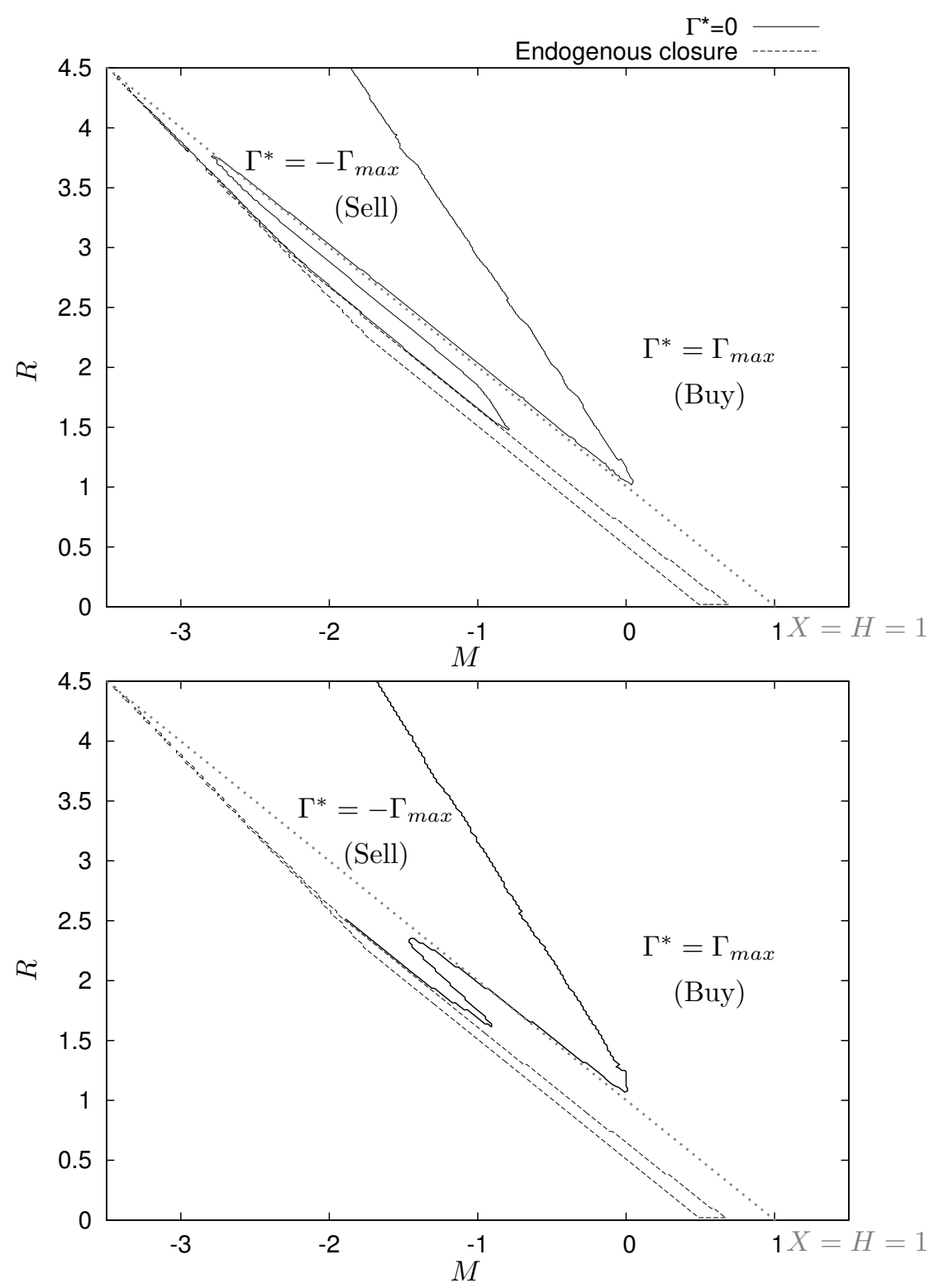

Fig. 1. Map plots of the $\Gamma^{*}$ decisions with restriction $\Gamma_{\max }=0.3$, for a CRRA utility function, at times $t=0.8$ (top), and $t=0$ (bottom), where $\Gamma^{*}=-\Gamma_{\max }$ implies selling shares of risky investment and $\Gamma^{*}=\Gamma_{\max }$ implies buying shares. The region enclosed by dashed lines represents endogenous closure. The gray dotted line corresponds to the high-water mark.

liquidity restriction affects more the rate at which the manager's position approaches the optimal than the optimal position itself. We stress that the importance of figure 2 is the reduction of leverage risk due to liquidity and not the shape of the curve 


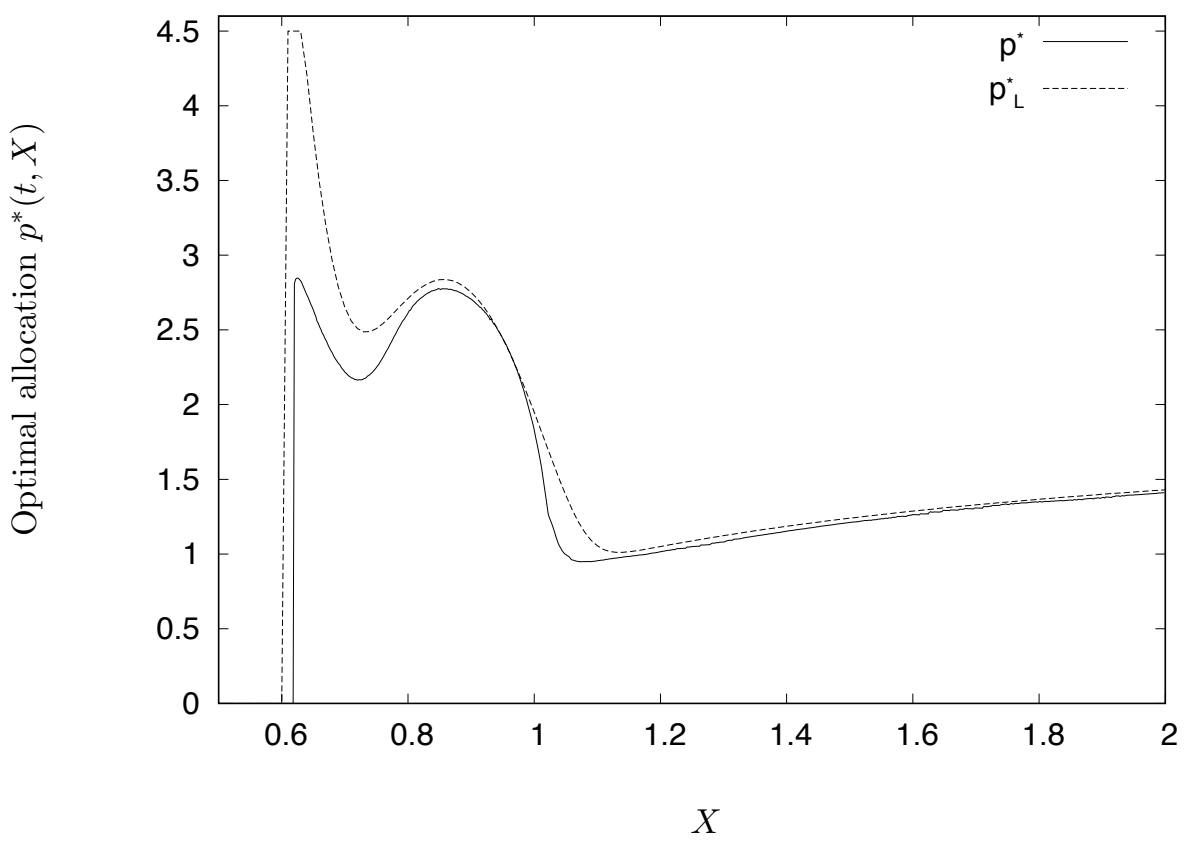

Fig. 2. Comparative effect of the manager's optimal allocation with a liquidity constraint (named $p^{*}$, solid line) and the fully liquid optimal allocation (named $p_{L}^{*}$, dashed line) at time $t=0$, and $\Gamma_{\max }=0.3$.

which is akin to that of Hodder \& Jackwerth (2007). Additionally, both curves seem to converge in the ramps to Merton's optimal (for $X>1.2$ ), because the strategies in these positions are more conservative and reduce the leverage risk.

\subsection{Liquidity effects on the utility of wealth}

To show the effect the liquidity constraint has on the manager's value function (i.e. expected utility of wealth), we fixed the fund value at $X=0.75$ and plot the value function at time $t=0$ changing the liquidity constraint parameter $\Gamma_{\max }$. Figure 3 illustrates the value function $J$ for a small fraction of the range around the optimal allocation $p^{*}(t, X) \approx 2.1$ (respectively $R \approx 1.6$ ) so that we can see better the shape of the curve. As the restriction becomes more severe (i.e. smaller values of $\left.\Gamma_{\max }\right)$, the values of $J$ decrease proportionally with the distance to the maximum point, implying that the restriction affects more the values farther from the optimal allocation.

Motivated by this, we define the cost of being away from the optimal position by $\mathcal{C}$ as the difference between the certainty equivalent values having different $\Gamma_{\max }$ restrictions (the certainty equivalents values are the inverse values of the value function, and these are feasible to calculate since we use a CRRA utility function). So $\mathcal{C}$ represents how much the manager is losing when investing in more illiquid 


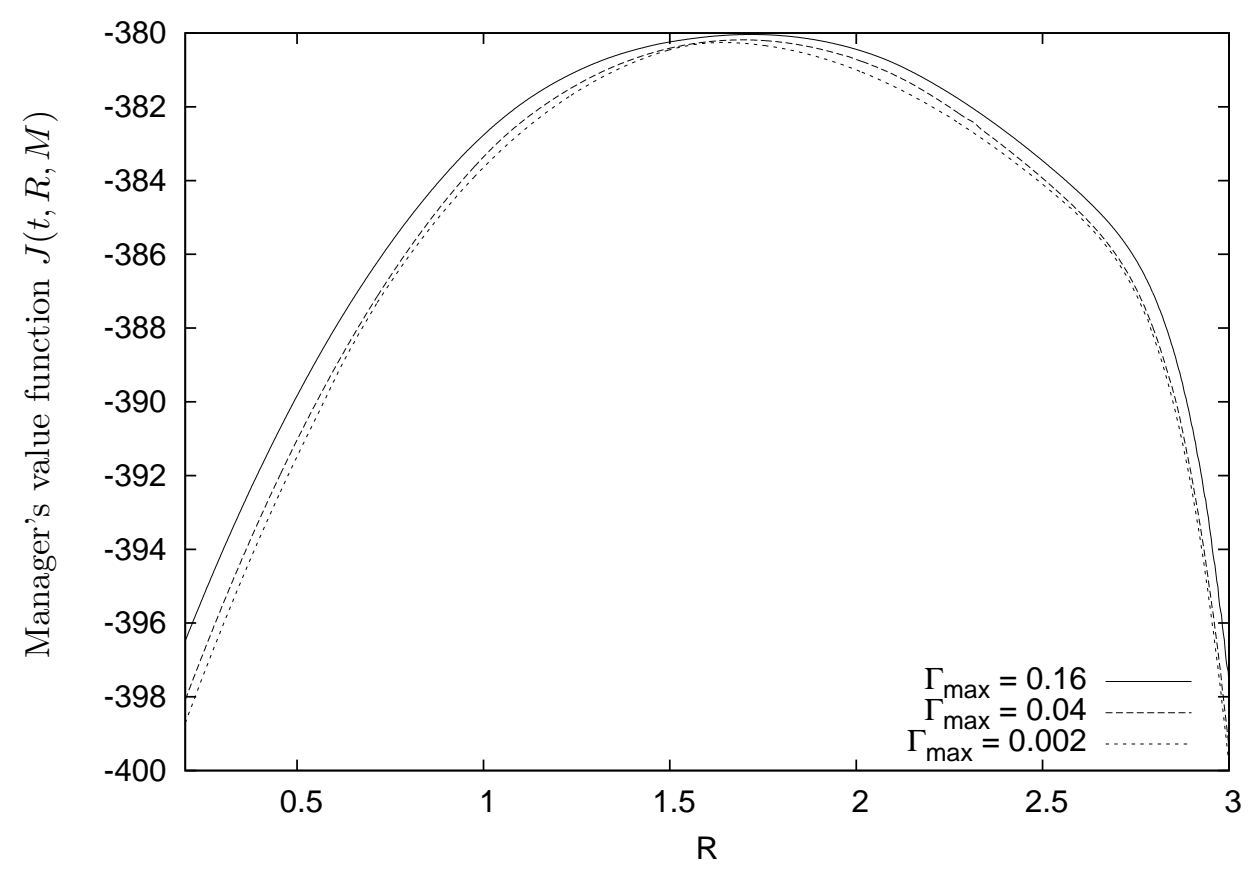

Fig. 3. The value function $J(t=0, R, M)$ for fixed wealth $X=0.75$, with liquidity restrictions $\Gamma_{\max }=0.16,0.4$ and 0.002 .

assets, when all other parameters remain the same, that is

$$
\mathcal{C}\left(\Gamma_{1}, \Gamma_{2}, R, M\right)=U^{-1}\left(\left.J(0, R, M)\right|_{\Gamma_{\max }=\Gamma_{1}}\right)-U^{-1}\left(\left.J(0, R, M)\right|_{\Gamma_{\max }=\Gamma_{2}}\right) .
$$

In figure 4, we plot the cost function $\mathcal{C}$ for fixed fund values $X=0.75$ (below $H=1$ ) and $X=1.2$ (above $H$ ), and through different portfolio choices $p$, where we set $\Gamma_{1}=0.16$ as the benchmark. As a result, figure 4 shows that the highest cost corresponds to funds with high leverage levels, i.e. $p>3.5$. This is because the fund can have a negative variation (i.e. a drop in the value of the risky asset in an instant of time), and this variation takes a manager in a high leverage position to a position leveraging more than the maximum permitted (and hence incurs on penalties or even in the closure of the fund). In our case leveraging more than 4.5 means closure of the fund and therefore all values close to (but less than) 4.5 have a higher cost. In our simulation, these values agree in $p=4.5$ because of the closure condition. Additionally, figure 4 shows that, as the fund value decreases, the cost in non-leveraged positions (i.e. $p<1$ ) increases (as we can see in the top graph), which agrees with figure 2 , since funds with values less than unity (i.e. $X<1=H$ ) have optimal positions with riskier portfolios than wealthier funds (i.e. $X>1$ ). Also for the plot corresponding to functions having $X=1.2$ (bottom graph), we can see that the cost for values of $p<3$ does not change dramatically, and is less 

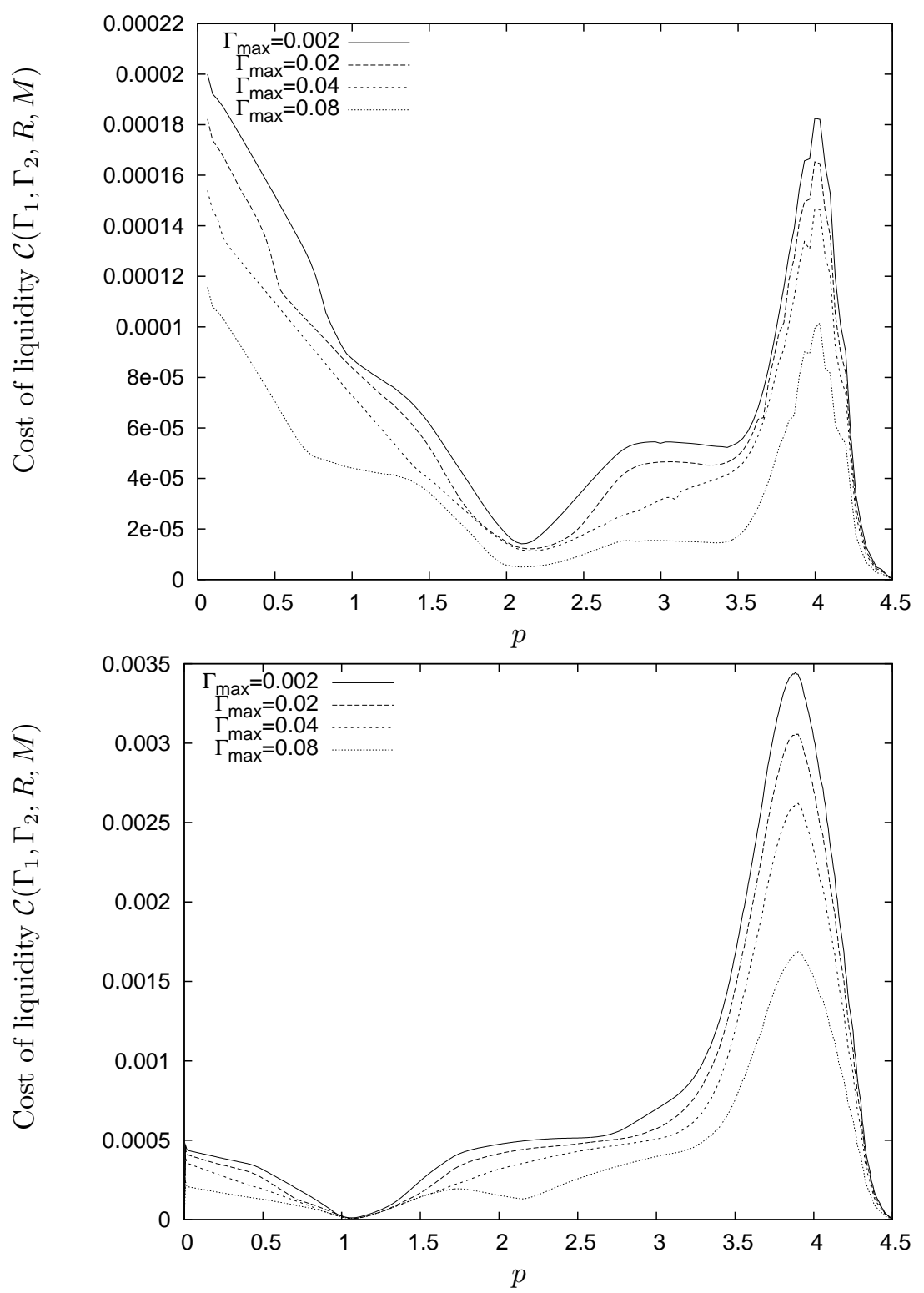

Fig. 4. Cost due to liquidity constrained functions for fixed fund values, $X=0.75$ on the top and $X=1.2$ on the bottom. The cost is calculated by subtracting certainty equivalent values with different liquidity constraints $\Gamma_{2}=0.08,0.04,0.02$ and 0.002 from the value function with liquidity constraint $\Gamma_{1}=0.16$.

significant at $p \approx 1.1$, which is the optimal position for $X=1.2$. So the cost of being below the optimal position affects more managers who have not achieved the high-water mark, i.e. those who have not achieved the incentive fee. 
For example, figure 4 shows that at the level of wealth $X=0.75$, the maximum cost of being away of the optimal (due to the liquidity) is about $\mathcal{C}=0.0002$, which means that for an investment of $\$ 750,000$ the cost (or deduction from the manager's payment) would be $\$ 200$. In the same manner, from the bottom graph we deduce that for an investment of $\$ 1^{\prime} 200,000$ the maximum cost would be $\$ 3,500$, note that the restriction varies from being able to trade a maximum of $\$ 160,000$ per year to a maximum of $\$ 2,000$ per year.

\subsection{More severe liquidity restriction with higher expected returns}

Next, we explore the scenario where the manager invests in more illiquid assets in order to obtain more return (or alpha) from these assets. To do so we compare a hedge fund having restriction $\Gamma_{\max }=0.3$ with the same fund but having half of the restriction $\frac{1}{2} \Gamma_{\max }=0.15$ (implying a more severe restriction). As expected, if the rate of return for the risky asset $\mu$ remains the same for both funds the value function with the more severe restriction $\left(\frac{1}{2} \Gamma_{\max }\right)$ have a lesser value. Thus we explore the consequences of increasing the value of the expected rate of return $\mu$ (and correspondingly $\sigma$ to keep Merton's optimal portfolio $\mathfrak{p}$ constant. We decide to keep this $\mathfrak{p}$ constant because it resembles the Sharpe Ratio in the sense that it is measuring the excess of return $\mu-r$ relative to volatility $\sigma^{2}$ and risk-aversion $\gamma$, in order to have comparable portfolios. We find that having this new higher return but less liquid asset makes value function increase as well, although contrary to intuition the value function (and hence the certainty equivalent) does not increase proportionally for all the values of the fund. In figure 5 we show the certainty equivalent values of manager's wealth for the fixed wealth value $X=1.1$ having the original restriction of $\Gamma_{\max }=0.3$ (and original set of parameters, as in table 4) compared to the certainty equivalent having half of this restriction but increasing the expected rate of return to $\hat{\mu}=0.1$, to resemble the higher return (i.e. $\hat{\mu}>\mu$ ). We observe in this figure that the certainty equivalent value for the fund with more severe restriction (and hence with higher expected rate of return) has less payoff in the more leveraged positions, although it has higher payoff in the positions with less risk, i.e. smaller $p$. This is a direct consequence of the liquidity constraint and may be observed from this results that, with a more severe restriction, optimal portfolios should be moved away from leverage.

To complement these results, in figure 6 we show the difference between the two certainty-equivalent payoffs for the manager, and we see that the negative values correspond to the region where investing in a more restricted asset is more beneficial because of the higher returns, and the positive values correspond to the region where investing in less-restricted assets is better because of the leverage (riskier) position taken by the manager. Figure 6 includes the plots for different values of wealth $X=0.8,0.9,1,1.1$ and 1.2 , and we observe that the positive value region grows larger as the fund value $X$ increases. This is because as the fund's wealth increases 


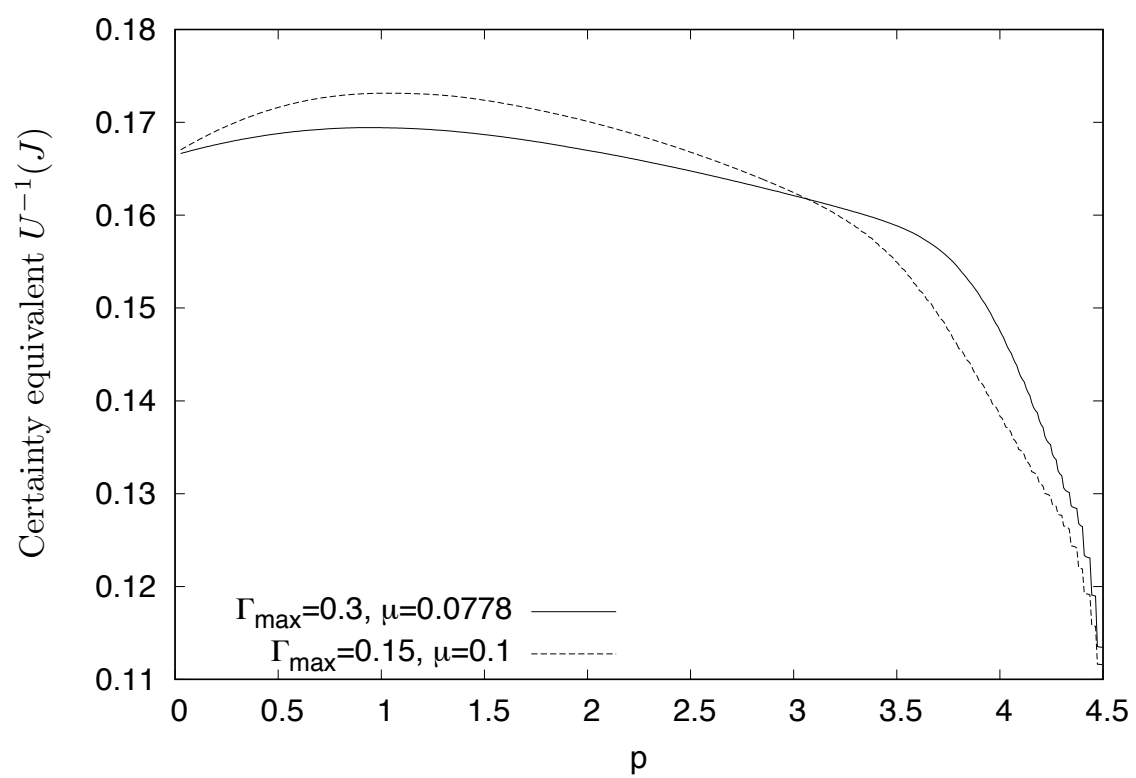

Fig. 5. Comparison of the certainty equivalent values for the fixed fund value $X=1.1$, where the $\Gamma_{\max }$ restriction is changed and accordingly the expected rate of return $\mu$ is changed.

(specifically above the high-water mark) the manager prefers safer investments and since the positive values occurs in the more leveraged positions $(p>2)$, the manager prefers investments with a lower liquidity restriction (higher value of $\Gamma_{\max }$ ) to be able change his position fast enough to a safer position (by moving to a lower value of $p$ ).

\section{Multiperiod case}

In this section, we extend the model discussed previously to allow it to incorporate multiple periods. In particular, we reset the high-water mark, say, yearly (for example). Since the same model holds, we show how to extend our numerics and describe numerical results. Furthermore, comparisons with the multiperiod model shown in Hodder \& Jackwerth (2007) are given. Our approach to model multiple periods differs from Lan et al. (2013), Goetzmann et al. (2003) and Guasoni \& Obłój (2013), because we use a discrete maximum of the fund value $X_{t}$ (maximum value over a discrete set of times), as the resetting value for the high-water mark, rather than using the continuous maximum of the fund value, which we believe is a more realistic setting. Therefore, the manager's optimization problem now includes a high-water mark variable which may change at discrete times, as is explained next. 


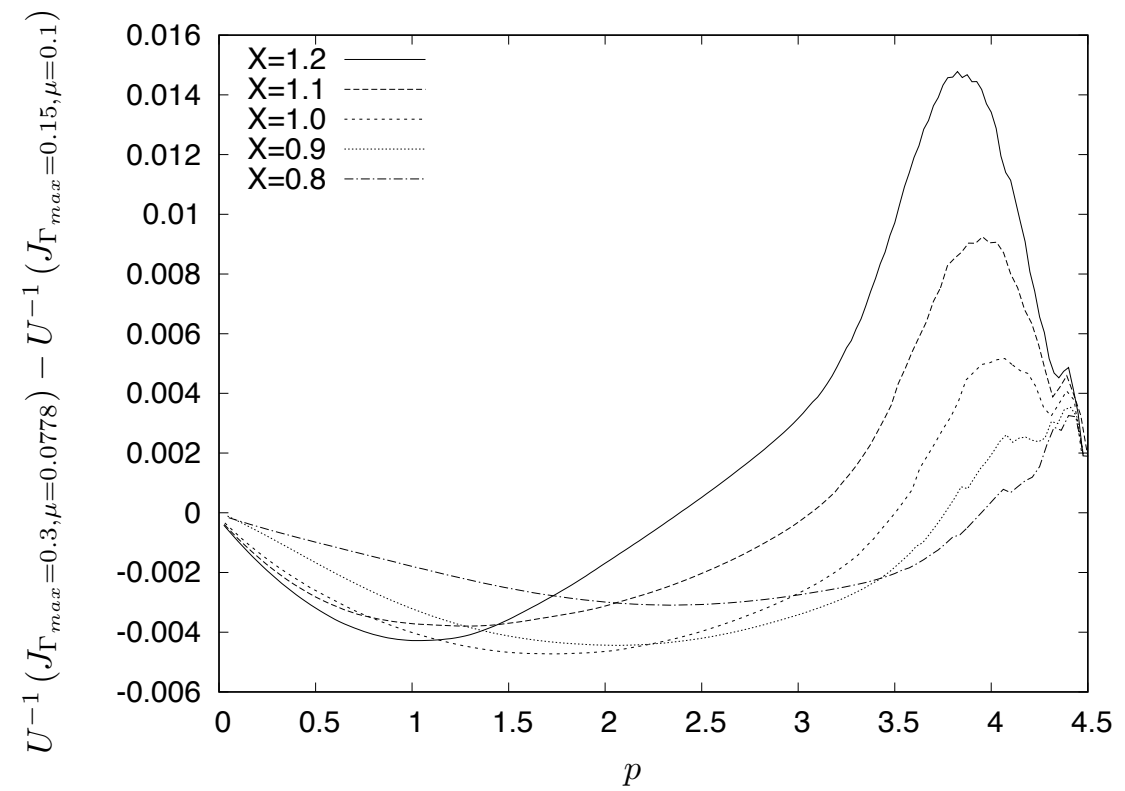

Fig. 6. Differences of the certainty equivalent manager's wealth comparing the liquidity restrictions and rates of return $\Gamma_{\max }=0.3, \mu=0.0778$ with $\Gamma_{\max }=0.15, \mu=0.1$, for different fixed fund values.

\subsection{Model extension}

Let us first explain what happens at the end of a year (period) for a multiple-year hedge fund. Suppose ending-year times are $T_{1}, T_{2}, \ldots T_{n}$ so that year $i$ ends at time $T_{i}$, these times are also known as sampling times. Under our assumptions, at the end of the year, the manager receives payment for managerial and incentive fees. After the manager gets paid, the high-water mark is reset (if the fund's value is higher than $H$ ), and then the next year is treated as a single year until the next year's end, that is to say between sampling times the fund's behaviour is the same as the single period case. Note that, in this case, the payment of the incentive is made at discrete and specific times, andd it is not paid at any time the manager surpasses the high-water mark, as in Guasoni \& Obłój (2013) and in Lan et al. (2013).

Since the high-water mark is, generally, reset at each year period, then our model changes in the sense that $H$ is no longer a constant but a variable depending on the fund's value and time. That is, at the end of the time period $\left[T_{i-1}, T_{i}\right)$ we have two cases for the high-water mark:

- If the performance of the fund is positive, the fund value is greater than the previous high-water mark (i.e. $X_{T_{i}} \geq H_{i-1}$ ); thus the high-water mark is reset, that is $H_{i}:=X_{T_{i}}$.

- If the fund's value is below the previous high-water mark (i.e. $X_{T_{i}}<H_{i-1}$ ) then 
the high-water mark is not reset $H_{i}:=H_{i-1}$.

Specifically, given the dates of evaluation $\left\{T_{1}, T_{2}, \ldots T_{n}\right\}$, the high-water mark at time $t$ prior expiry (i.e. $T_{k} \leq t<T_{k+1}$, for some $0 \leq k<n$ ) is

$$
H_{k}=\max \left\{X_{T_{i}}: i=0, \ldots, k\right\}
$$

where we assume the initial high-water mark $H=1$ (the high-water mark for the first time period, i.e. $T_{0}=0$, which is related to the initial value of the fund). This behavior of the high-water mark over the years can be treated as a discrete time look-back option, hence the value function $J$ now depends on the extra variable $H$ (i.e. $J(R, M, H, t))$.

At sample time $T_{i}$, the values of $X_{T_{i}^{+}}$greater than $H_{i}$ should not exist. Thus, for any point satisfying $X_{T_{i}^{+}}>H_{i}$, we change the value function with the value function having the correct high-water mark, i.e. the value function $J\left(R_{T_{i}}, M_{T_{i}}, H_{i-1}, T_{i}\right)$ must be replaced by $J\left(R_{T_{i}}, M_{T_{i}}, X_{T_{i}^{+}}, T_{i}\right)$, where $H_{i}=X_{T_{i}^{+}}=R_{T_{i}^{+}}+M_{T_{i}^{+}}$. The next step in this setting is to add the payment of the managerial fees.

Let us assume that the value function is given in terms of the manager's wealth and the high-water mark (we suppose that $\hat{J}\left(W_{t}, H\right)=J\left(R_{t}, M_{t}, H, t\right)$ ), and we add the fees' payment at time $T_{i}$, then we must have the following

$$
\hat{J}\left(W_{T_{i}}, H_{i}\right):=\hat{J}\left(W_{T_{i}}+F P\left(X_{T_{i}}, H_{i-1}\right), X_{T_{i}^{+}}\right),
$$

where

$$
F P\left(X_{T_{i}}, H_{i-1}\right)=(1-a) b X_{T_{i}}+(1-a) c\left(X_{T_{i}}-H_{i-1}\right)^{+}
$$

represents the fee payment, which is added to the manager's wealth. Notice that we write $\hat{J}$ for the value function, but in terms of $W$, i.e. the manager's wealth.

Then, in the case that $X_{T_{i}^{+}}<H_{i-1}$ there is nothing to do but pay fees and continue (We do not reset $H_{i-1}$ ), that is

$$
\hat{J}\left(W_{T_{i}}, H_{i}\right):=\hat{J}\left(W_{T_{i}}+F P\left(X_{T_{i}}, H_{i-1}\right), H_{i-1}\right) .
$$

Since the payment of the manager's fees is taken from the the fund, we have that at sample time $T_{i}$, the fund value has a jump condition because of the payment of the fees to the manager, so we have that

$$
X_{T_{i}^{+}}=X_{T_{i}^{-}}-F P\left(X_{T_{i}^{-}}, H_{i-1}\right)
$$

and immediately after this payment is made we may reset the high-water mark to

$H_{i}=\max \left\{H_{i-1}, X_{T_{i}^{+}}\right\}$. Note that we actually use equation (5.2) where $X_{T_{i}^{+}}$is as in (5.5).

\subsection{Multiperiod Numerical approach}

In this section we describe the numerical techniques needed to extend the approach given in section 5.1, therefore we are only concerned with the sampling times. 
Suppose that the final time is $T=T_{n}$, so the computation starts with final condition

$J(R, M, H ; T)=U\left(a(R+M)+(1-a) b\left(T-T_{n-1}\right)(R+M)-(1-a) c(R+M-H)^{+}\right)$

and solve this problem in the same way as for a single period up to $T_{n-1}$, i.e. this means to consider $H$ as constant through the interval $\left[T_{n-1}, T_{n}\right)$. At time $T_{n-1}$, we continue with the payment of the fees as follows

$$
J_{t o}\left(R, M, H_{n-1}, T_{n-1}\right)=J\left(R_{F P}, M_{F P}, H_{n}, T_{n-1}\right),
$$

where $H_{n}=\max \left\{H_{n-1}, X-F P\left(X_{T_{n-1}}, H_{n-1}\right)\right\}, X_{T_{n-1}}=R_{T_{n-1}}+M_{T_{n-1}}$, the fee's payment $F P\left(X_{T_{n-1}}, H_{n-1}\right)$ is as in (5.3), and $R_{F P}, M_{F P}$ represent the amount of money left in the risky and risk-less technologies after paying fees respectively. We assume that the manager receives his payment in assets and money. Then regarding $\rho$ as the proportion taken from the risky asset to pay the fees, we have $R_{F P}=$ $R-\rho F P\left(X, H_{n-1}\right)$ and $M_{F P}=M-(1-\rho) F P\left(X, H_{n-1}\right)$. In the case where $X_{T_{i}}>H_{i}$, since the $H_{i}$ has just been reset, the value of $X$ cannot be greater than the new high-water mark (i.e. the maximum), thus we must obtain the value from the corresponding value according to the high-water mark reset, that is $H_{n}=$ $X-F P\left(X_{T_{n-1}}, H_{n-1}\right)$.

Note that equation (5.7) only represents the value function for a manager where the fund value has decreased to $R_{F P}+M_{F P}$, which it is useful as a take-off point (denoted by $J_{t o}$ ). Then, to add the actual fee payment to the manager's wealth (in the value function $J$ ), we use the certainty equivalent value. Specifically, we add the fee payment to this certainty equivalent value and calculate the corresponding utility of this value, that is the value function at $T_{n-1}$ corresponds to

$$
\left.J\left(R, M, H, T_{n-1}\right)=U\left(F P\left(X, H_{n-1}\right)\right)+U^{-1}\left(J_{t o}\left(R, M, H, T_{n-1}\right)\right)\right) .
$$

Therefore, we solve the multiperiod by continuing with this calculations at sampling times up to $t=0$ and using the single period solution between sampling times.

\subsection{Multiperiod Results}

In this section, we show results for the multiperiod case and as a benchmark we use the results presented in Hodder \& Jackwerth (2007), and we focus on the differences between their model and our model.

\subsubsection{Hodder and Jackwerth scheme}

In Hodder \& Jackwerth (2007), we find a model without liquidity constraints, thus they model the fund's wealth $X$ as a single variable. They distinguish between the risky and risk-free investments by letting $p X$ be the risky investment and (1$p) X$ be the risk-less investment and they control the value of $p$ (i.e. the portfolio), respectively our model separates $R=p X$ and $M=(1-p) X$. In their approach, the 
value $X$ does not represent the fund value but the fund value relative to the highwater mark, and all other parameters are also relative to $H$. By this we mean that they work under the change of variables $\tilde{X}=\frac{X}{H}$ and all the values are regarded as being scaled to the high-water mark, this change is made for simplicity of the model specially when evaluating multiple periods of time (to keep $H$ constant through the different periods). Our approach is more realistic in the sense that it does not work with relative values but instead with absolute values, and we overcome the difficulties in Hodder \& Jackwerth (2007) by adding an extra dimension to our PDE and solve it using the numerics described earlier. On the other hand, Hodder \& Jackwerth (2007) use a scheme that is a discrete dynamic programming problem, which is similar to the quadrature method (for more information about the quadrature method of Andricopoulos et al. (2003)).

\subsection{Multiperiod with liquidity constraint}

To work with the actual values of the fund $X$ (represented by $R$ and $M$ ), we need to add the extra dimension on $H$, meaning that the path dependence is modeled as an extra dimension.

Since the fees are paid from within the fund, we have to calculate the take-off point as the point corresponding to the hedge fund's wealth after the fees are paid. This value is not unique since it lies on the diagonal line $R+M=X-F P(R, M, H)$. Therefore we calculate for the pair $(R, M)$ the corresponding proportional take-off value $\left(R^{*}, M^{*}\right)$ on the line $R^{*}+M^{*}=X-F P(R, M, H)$. By proportional, we mean that we do not alter the fund's distribution after the fees are paid $(\rho=p)$, i.e. the proportion invested in risky and risk-free assets remains the same. Hence we must:

(1) Calculate the proportional take off points for the balancing

$$
\begin{aligned}
R^{*} & =R-\rho F P(R, M, H), \\
M^{*} & =M-(1-\rho) F P(R, M, H)
\end{aligned}
$$

and then interpolate to obtain the value function $J\left(R^{*}, M^{*}, H^{*}, t_{i}\right)$, where $H^{*}=$ $\max \left\{H, R^{*}+M^{*}\right\}$.

(2) Calculate the new final value with the certainty equivalent having updated high-water mark, that is

$$
J\left(R, M, H, t_{i}^{-}\right)=U\left(F P(R, M, H)+U^{-1}\left(J\left(R^{*}, M^{*}, H^{*}, t_{i}\right)\right)\right) .
$$

Then, the previous numeric approach is then applicable.

In figure 7, we show a comparison between the fully liquid hedge fund, similar to Hodder \& Jackwerth (2007), (on the left graph) and the version with liquidity constraint (on the right graph), and we show the dynamic strategies from three years to two years prior to the terminal time, i.e. from $t=27$ to $t=28$ (for $T=30$ ). We can see in this figure that the maximum leverage (near the endogenous liquidation at about $X=0.55)$ in the fully liquid scenario has $p_{L}^{*} \approx 4.5$ and with 


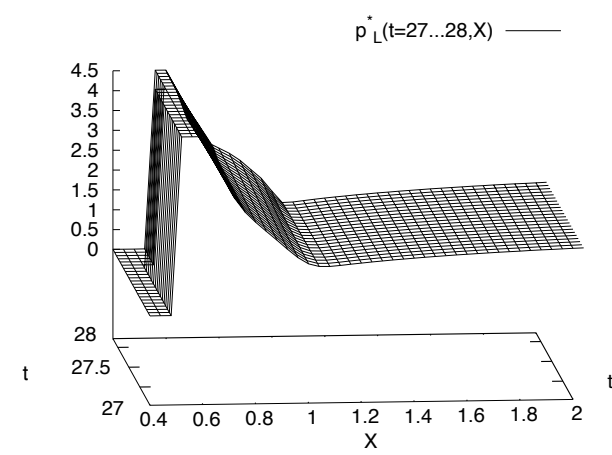

(a) Fully liquid

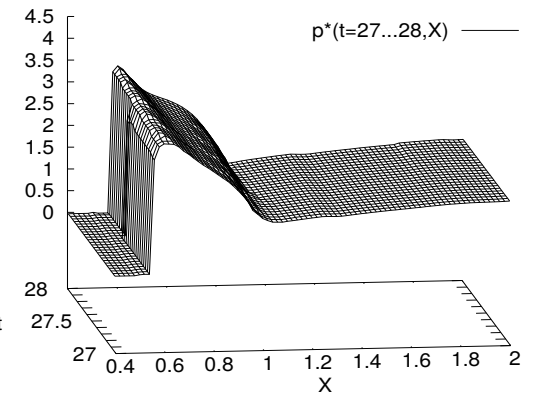

(b) Liquidity constrained

Fig. 7. Surface plots $\left(t \times X\right.$ vs $\left.p^{*}\right)$ for the manager's optimal allocation position, $p_{L}^{*}(t, X)$ for the fully liquid case (left graph) and $p^{*}(t, X)$ for the case with liquidity restriction (right graph), in the time period $t \in[27,28]$ for $T=30$.

liquidity constraint has about $p^{*} \approx 3.5$. Therefore, in the presence of a liquidity constraint, the manager takes less risks, specially near the endogenous closure, due to the additional inherent risks of illiquidity. For times farther away from closure, the peak of higher risks near the endogenous closure is reduced in both cases to the point that its effect is insignificant (about 10 years prior to closure, i.e. $T-10$ ). It is to remark that, in view of the liquidity constraint, the manager reduces the portfolio risk with less leveraged positions, but this only happens near the closure region, which implies that the manager does not want to risk closing the fund.

\subsubsection{Paying the managerial fees in cash}

It is not always the case where the manager of the fund is paid proportional in shares and in money (i.e. cash), in such a way that the fund's portfolio does not get altered, as is the previous section. It could be the case that the mechanism to pay the manager is only money, only shares or any combination of those.

Next, we look at the difference in portfolio allocations by changing the proportion in which the manager is paid, i.e. between Hodder \& Jackwerth (2007) multiperiod replication but adding a liquidity constraint where manager's fees come from an outside source, the multiperiod model paying the manager fees from the fund proportionally in assets and in cash (as in the previous subsection), and the multiperiod model paying the manager fees in cash (from the fund's bank account), this last one corresponds to

$$
J\left(T_{i}^{-}, R, M, H\right)=U\left(F P(R, M, H)+U^{-1}\left(J\left(T_{i}, R, M^{*}, H^{*}\right)\right)\right),
$$

where $H^{*}=\max \left\{H, R+M^{*}\right\}$ and $M^{*}=M-F P(R, M, H)$, i.e. $\rho=0$ in (5.9) and (5.10).

For this case, we set $T=3$ and sampling times $T_{1}=1, T_{2}=2$. In figure 8 , 


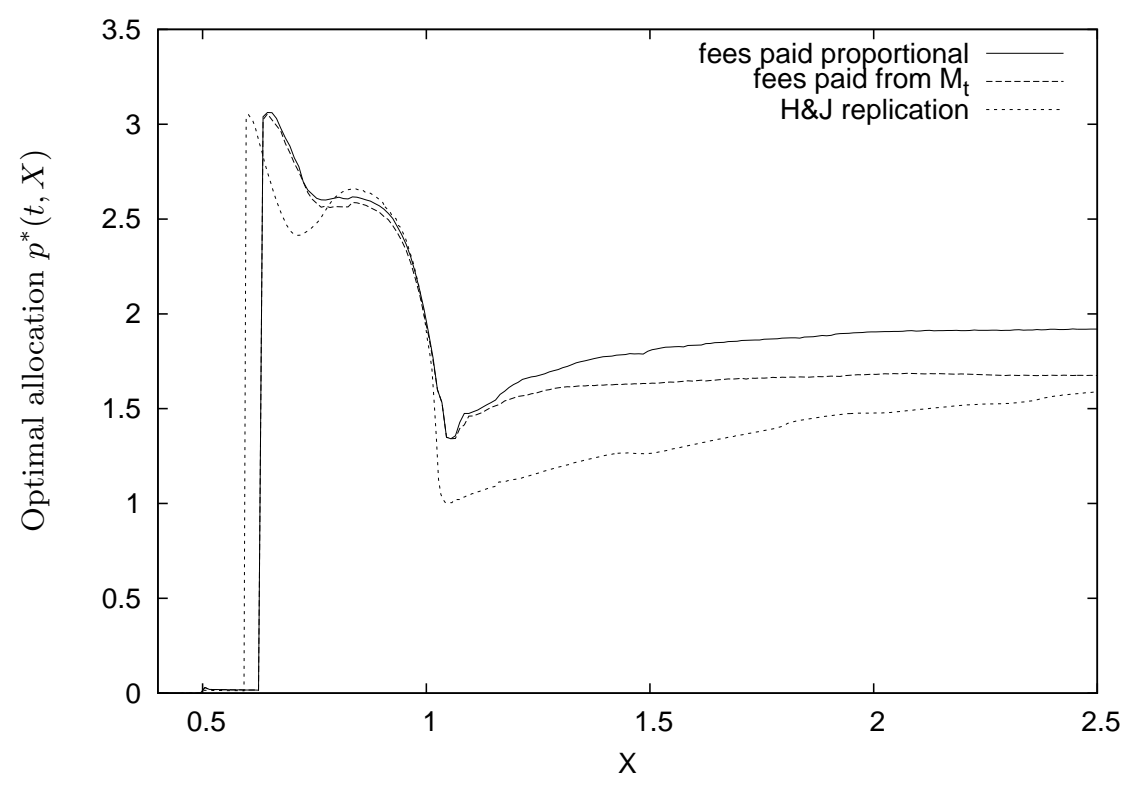

Fig. 8. Optimal allocation $p^{*}$ for $t=T-1.3$, where the manager fees are paid from the money account $M_{t}$, proportional in shares and money, compared with Hodder and Jackwerth replication with liquidity constraint.

we plot the manager's optimal allocation for a fixed time between $T_{1}$ and $T_{2}$, i.e. $t=1.7$ or $T-1.3$. We note that the difference between paying the fees proportionally and from the bank account $M_{t}$ (i.e. in cash), is that in the latter the manager is less aggressive for fund values above the high-water mark $H=1$ and as the fund value increases it stabilises at $p^{*} \approx 1.65$, which is less than Merton's optimal value $(\mathfrak{p}=2)$. Whereas paying the fees proportionally induces the manager to replicate Merton's optimal position for $X$ sufficiently large. Furthermore, we find a different behaviour in our version of Hodder \& Jackwerth (2007) with liquidity constraint because for values of the fund $X$ above $H$, the manager takes less risk due to the balancing condition (5.11).

For fund values $X<H$, we see a similar behavior as that described for $X>$ $H$. That is, when the fees are paid proportionally, the manager takes more risk, since after the payment of the fees, the fund has less value $(R+M-F P(R, M)$ or $X-F P(X))$ which could lead to closure. This can be clearly seen in figure 9 , where we plot the manager's optimal allocation position at time $t=0.7$ (i.e. $t=T-2.3$ ), and again suggesting that when the fees are paid proportionally the manager behaves more aggressively.

From figures 9 and 8 , we note that funds with liquidity restrictions converge, when $X$ grows large, to a value smaller than Merton's optimal strategy $\mathfrak{p}$. In the fully liquid case, as $X \rightarrow \infty$, the strategy converges to Merton (i.e. $\mathfrak{p}=2$ ) as in Hodder \& Jackwerth (2007), but with the liquidity constraint this is no longer true 


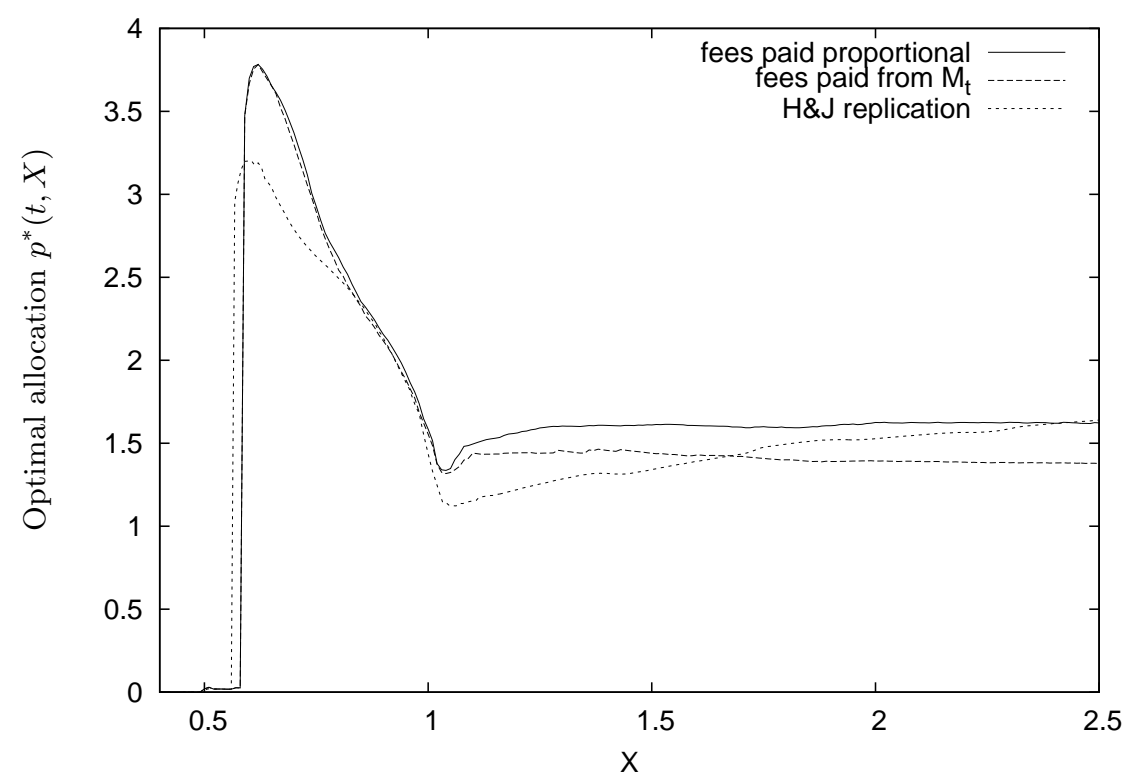

Fig. 9. Optimal allocation $p^{*}$ for $t=T-2.3$, where the manager fees are paid from the money account $M_{t}$, and proportional in shares and money account, and in Hodder and Jackwerth replication with liquidity constraint.

and under a liquidity restriction, therefore Merton's theory should be regarded more carefully.

\section{Conclusions}

In this paper, we present an original model for hedge-fund management with a liquidity constraint. The modelling involves combining the work of Longstaff (2001), Hodder \& Jackwerth (2007) and that of Guasoni \& Obłój (2013). We use stochastic control techniques, which results in a value function with three state variables $(R$ representing the amount of money invested in a risky asset, $M$ representing the amount of money in a bank account and $H$ the high-water mark) plus time, where the liquidity constraint is given by restricting the amount of money $\Gamma$ that can be traded between $R$ and $M$ in a small period of time $\Delta t$ (namely $\Gamma \in\left[-\Gamma_{\max }, \Gamma_{\max }\right]$ ). Under this setting we assert uniqueness of the solution (by using Crandall-Ishii and Lions' lemma) and we use the modeling tools for American and Look-Back options to represent the behavior of the endogenous closure and the high-water mark resetting, respectively. Modeling the actual amounts of money in the risky and risk-less investments lets us utilize a novel numerical approach for solving this class of problems in a way that is numerically tractable with finite differences, that is using a combined Semi-Lagrangian Crank-Nicolson PSOR scheme.

The first results in this paper tells us how to trade, depending on the time-state 
position, which is comparable with Hodder \& Jackwerth (2007), although we are able to establish a less risky trading behavior of the manager in the presence of liquidity constraints. Specifically, that the manager lowers risk-exposure at endogenous closure. Thereafter, because managers have to change dynamically their positions to stay in the optimal allocation, we measure the cost of being at a sub-optimal allocation position as the difference between the certainty equivalent values having different liquidity restrictions and we observe that this difference is more profound in the leveraged zones and in funds that have not achieved the incentive fee $(X<H)$.

We reaffirm the common knowledge statement that managers opt to invest in assets with higher expected rates of return but are less liquid because they produce more returns although in our results the manager does not always benefit from these investments. This is because the lack of liquidity incurs in additional risks and therefore leveraged positions should be taken more carefully.

Finally we study the manager's optimal allocation in multiple years, modeling the high-water mark as a discrete maximum and hence inheriting the numerical treatment of look-back options. The multiperiod results reinforce our theory that the manager acts less aggressively and takes less risks with liquidity constraints, compared with the fully liquid case. Our model also lets us extend the results and check the differences between paying the manager in cash and paying the manager proportionally between cash and assets, and we find that the manager takes more risks in the latter scenario.

Since hedge funds are unregulated investments, it is a common practice that managers of hedge funds do not report all of their movements and the investor (most likely) does not know about managers' strategy. Hence there is an unexplored area of research on the consequences of having partial information or partial hedging by investors (and managers as well) in hedge funds. Since having only partial information could lead to incomplete markets within a non-Markovian setting, this would necessitate different mathematical techniques such as Malliavin calculus tools, and hence would lead to a whole new model/project. In the literature, there has already been some work capturing similar characteristics to this: Menoukeu Pamen et al. (2013) derive a general stochastic maximum principle under partial information and Di Nunno \& Øksendal (2009) consider the optimal portfolio problem for a manager who does not have full access to market information, and we believe this theory could be extended to hedge funds.

Clearly there is still much work to be done on modeling hedge funds and this will become more important in the future as investors and fund managers search for the highest returns and lowest risk in an increasingly uncertain world.

\section{Appendix A. Unique Solution}

Following the lines of Touzi (2013), we know that the value function $J$ is a viscosity solution and for a uniqueness result we have to check, for a functional $F$, the following assumptions: 
(1) $\exists \vartheta>0$ such that

$$
F(t, X, r, v, \vec{q}, \mathbf{A})-F\left(t, X, r^{\prime}, v, \vec{q}, \mathbf{A}\right) \geq \vartheta\left(r-r^{\prime}\right) .
$$

For all $r \geq r^{\prime}$, and $(t, X, v, \vec{q}, \mathbf{A}) \in Q \times \mathbb{R} \times \mathbb{R}^{n} \times \mathcal{S}_{n}$.

(2) There exists $\bar{w}: \mathbb{R}^{+} \rightarrow \mathbb{R}^{+}$with $\bar{w}\left(0^{+}\right)=0$, such that

$$
F(t, y, r, v, \alpha(x-y), \mathbf{B})-F(t, x, r, v, \alpha(x-y), \mathbf{A}) \leq \bar{w}\left(\alpha|x-y|^{2}+|x-y|\right) .
$$

For all $(t, x),(t, y) \in Q, r \in \mathbb{R}$ and $\mathbf{A}, \mathbf{B}$ such that

$$
-\left(\frac{1}{\epsilon}+|\alpha|\right) \mathbb{I}_{2 n} \leq\left(\begin{array}{cc}
\mathbf{A} & 0 \\
0 & -\mathbf{B}
\end{array}\right) \leq\left(\alpha+\epsilon|\alpha|^{2}\right)\left(\begin{array}{cc}
\mathbb{I}_{n} & -\mathbb{I}_{n} \\
-\mathbb{I}_{n} & \mathbb{I}_{n}
\end{array}\right) .
$$

Particularly letting $\alpha=\frac{1}{\epsilon}$

$$
-3 \alpha \mathbb{I}_{2 n} \leq\left(\begin{array}{cc}
\mathbf{A} & 0 \\
0 & -\mathbf{B}
\end{array}\right) \leq 3 \alpha\left(\begin{array}{cc}
\mathbb{I}_{n} & -\mathbb{I}_{n} \\
-\mathbb{I}_{n} & \mathbb{I}_{n}
\end{array}\right) .
$$

Note that (A.4) implies that $\mathbf{A} \leq \mathbf{B}$ since the right side has norm 0 (the matrix norm in the usual sense $|\mathbf{A}|=\sup \{\mathbf{A} \eta \cdot \eta:|\eta|=1\})$.

Therefore, to prove uniqueness for viscosity solutions to the final value problem in equations (2.9) and (2.8). We let $x=\left(x_{R}, x_{M}\right)$ and $y=\left(y_{R}, y_{M}\right)$, then we may define the HJB functional as

$$
\begin{aligned}
F(t, x, J, & \left.\frac{\partial J}{\partial t}, \frac{\partial J}{\partial x}, \frac{\partial^{2} J}{\partial x^{2}}\right) \\
& =-\frac{\partial J}{\partial t}-\frac{1}{2} x \boldsymbol{\sigma} \frac{\partial^{2} J}{\partial x^{2}}(x \boldsymbol{\sigma})^{t r}-\max _{\Gamma}\left\{\left(\mu x_{R}+\Gamma(t), r x_{M}-\Gamma(t)\right) \cdot \frac{\partial J}{\partial x}\right\},
\end{aligned}
$$

where

$$
\begin{aligned}
\boldsymbol{\sigma} & =\left(\begin{array}{ll}
\sigma & 0 \\
0 & 0
\end{array}\right), \\
\frac{\partial J}{\partial x} & =\left(\begin{array}{ll}
\frac{\partial J}{\partial R}, \frac{\partial J}{\partial M}
\end{array}\right), \\
\frac{\partial^{2} J}{\partial x^{2}} & =\left(\begin{array}{cc}
\frac{\partial^{2} J}{\partial R^{2}} & \frac{\partial^{2} J}{\partial R \partial M} \\
\frac{\partial^{2} J}{\partial M \partial R} & \frac{\partial^{2} J}{\partial M^{2}}
\end{array}\right) .
\end{aligned}
$$

We notice that the function $F$ does not depend on $J(t, X)$ explicitly; a minor modification of the assumption is therefore needed. According to Crandall et al. (1992), notice that for every $\epsilon>0$ and viscosity subsolution $\tilde{J}$ of $(2.9), \tilde{J}_{\epsilon}:=\tilde{J}+\epsilon t$ is also a sub-solution of (2.9) since

$$
F\left(t, X, \tilde{J}_{\epsilon}, \frac{\partial \tilde{J}_{\epsilon}}{\partial t}, \frac{\partial \tilde{J}_{\epsilon}}{\partial X}, \frac{\partial^{2} \tilde{J}_{\epsilon}}{\partial X^{2}}\right) \leq-\epsilon<0 .
$$


Therefore, we obtain the result in (A.1) by choosing $\vartheta \leq \frac{1}{T}$ and using $\tilde{J}$ and $\tilde{J}_{\epsilon}$ since $\frac{\partial \tilde{J}_{\epsilon}}{\partial t}=\frac{\partial \tilde{J}}{\partial t}+\epsilon$.

If we let $Q=[0, T] \times O$, where $O$ is the state space, this modification is enough for the comparison theorem since by letting $\underset{\sim}{J} \in L S C(Q)$ and $\tilde{J} \in U S C(Q)$, we have that $\tilde{J}_{\epsilon}-\underset{\sim}{J} \leq \sup _{\partial Q}\left\{\tilde{J}_{\epsilon}-\underset{\sim}{J}\right\}$ and $\tilde{J}-\underset{\sim}{J} \leq \sup _{\partial Q}\{\tilde{J}-\underset{\sim}{J}\}+\epsilon T$.

The proof of the second assumption: For all $(t, x),(t, y) \in Q, r \in \mathbb{R}$ and $\mathbf{A}, \mathbf{B}$ such that

$$
-\left(\frac{1}{\epsilon}+|\alpha|\right) \mathbb{I}_{4} \leq\left(\begin{array}{cc}
\mathbf{A} & 0 \\
0 & -\mathbf{B}
\end{array}\right) \leq\left(\alpha+\epsilon|\alpha|^{2}\right)\left(\begin{array}{cc}
\mathbb{I}_{2} & -\mathbb{I}_{2} \\
-\mathbb{I}_{2} & \mathbb{I}_{2}
\end{array}\right) .
$$

Note that we have adapted the matrix to our case where the space dimension is 2 , and by letting $\alpha=\frac{1}{\epsilon}$ we obtain

$$
\left(\begin{array}{cc}
\mathbf{A} & 0 \\
0 & -\mathbf{B}
\end{array}\right) \leq 2 \alpha\left(\begin{array}{cc}
\mathbb{I}_{2} & -\mathbb{I}_{2} \\
-\mathbb{I}_{2} & \mathbb{I}_{2}
\end{array}\right)
$$

We can check this by changing the corresponding values of $F$, that is

$$
\begin{aligned}
F(t, y, r, v, \alpha(x-y), \mathbf{B})- & F(t, x, r, v, \alpha(x-y), \mathbf{A})= \\
-v & -\frac{1}{2} y \boldsymbol{\sigma} \mathbf{B}(y \boldsymbol{\sigma})^{t r}-\max _{\Gamma}\left\{\left(\mu y_{R}+\Gamma, r y_{M}-\Gamma\right) \cdot \alpha(x-y)\right\} \\
+ & +\frac{1}{2} x \boldsymbol{\sigma} \mathbf{A}(x \boldsymbol{\sigma})^{t r}+\max _{\Gamma}\left\{\left(\mu x_{R}+\Gamma, r x_{M}-\Gamma\right) \cdot \alpha(x-y)\right\} .
\end{aligned}
$$

Using that

$$
\begin{aligned}
\left.\frac{1}{2}\left((x \boldsymbol{\sigma}) \mathbf{A}(x \boldsymbol{\sigma})^{t r}-(y \boldsymbol{\sigma}) \mathbf{B}(y \boldsymbol{\sigma})^{t r}\right)\right) & =\frac{1}{2}\left((x, y)\left(\begin{array}{cc}
\boldsymbol{\sigma} & 0 \\
0 & \boldsymbol{\sigma}
\end{array}\right)\left(\begin{array}{cc}
\mathbf{A} & 0 \\
0 & -\mathbf{B}
\end{array}\right)\left(\begin{array}{ll}
\boldsymbol{\sigma} & 0 \\
0 & \boldsymbol{\sigma}
\end{array}\right)\left(\begin{array}{l}
x \\
y
\end{array}\right)\right) \\
& \leq \alpha\left((x, y)\left(\begin{array}{ll}
\boldsymbol{\sigma} & 0 \\
0 & \boldsymbol{\sigma}
\end{array}\right)\left(\begin{array}{cc}
\mathbb{I}_{2} & -\mathbb{I}_{2} \\
-\mathbb{I}_{2} & \mathbb{I}_{2}
\end{array}\right)\left(\begin{array}{ll}
\boldsymbol{\sigma} & 0 \\
0 & \boldsymbol{\sigma}
\end{array}\right)\left(\begin{array}{l}
x \\
y
\end{array}\right)\right) \\
& =\alpha \sigma^{2}\left(x_{R}-y_{R}\right)^{2} \leq \alpha \sigma^{2}\|x-y\|^{2}
\end{aligned}
$$

we obtain

$$
\begin{aligned}
& F(t, y, r, v, \alpha(x-y), \mathbf{B})-F(t, x, r, v, \alpha(x-y), \mathbf{A}) \leq \\
& \quad \alpha \sigma^{2}\|x-y\|^{2}-\max _{\Gamma}\left\{\left(\mu y_{R}+\Gamma, r y_{M}-\Gamma\right) \cdot \alpha(x-y)\right\}+\max _{\Gamma}\left\{\left(\mu x_{R}+\Gamma, r x_{M}-\Gamma\right) \cdot \alpha(x-y)\right\} .
\end{aligned}
$$

Now using that the maximum of $\Gamma$ is obtained over a compact set (In our case a bounded and closed segment), we denote the maximum for $x$ as $\Gamma_{x}^{*}$ and then

$$
\begin{aligned}
& F(t, y, r, v, \alpha(x-y), \mathbf{B})-F(t, x, r, v, \alpha(x-y), \mathbf{A}) \leq \\
& \quad \alpha \sigma^{2}\|x-y\|^{2}-\alpha\left(\mu y_{R}+\Gamma_{x}^{*}, r y_{M}-\Gamma_{x}^{*}\right) \cdot(x-y)+\alpha\left(\mu x_{R}+\Gamma_{x}^{*}, r x_{M}-\Gamma_{x}^{*}\right) \cdot \alpha(x-y),
\end{aligned}
$$

and thus

$$
F(t, y, r, v, \alpha(x-y), \mathbf{B})-F(t, x, r, v, \alpha(x-y), \mathbf{A}) \leq \alpha\left(\sigma^{2}+2 \mu\right)\|x-y\|^{2} .
$$


Then by letting $\bar{w}(z)=C z$, where $C>0$ is a constant greater than $\sigma^{2}-2 \mu$, we obtain the desired result in equation (A.2).

\section{Acknowledgments}

Hugo E. Ramirez thanks the collaboration of Carlos Alberto Castro, Rafael Antonio Serrano, at the Universidad del Rosario, for their valuable comments and to COLFUTURO/COLCIENCIAS call 568 for the economic support.

\section{References}

A. Andricopoulos, M. Widdicks, P. Duck \& D. Newton (2003) Universal option valuation using quadrature methods Journal of Financial Economics 67 (3), 447-471.

G.O. Aragon (2007) Share restrictions and asset pricing: Evidence from the hedge fund industry Journal of Financial Economics 83 33-58.

A. Buraschi, R. Kosowski \& W. Sritrakul (2014) Incentives and Endogenous Risk Taking: A Structural View on Hedge Fund Alphas Journal of Finance 69 (6), 2819-2870.

A. Cartea \& S. Jaimungal (2015) Optimal execution with limit and market orders Quantitative Finance 15 (8), 1279-1291.

U. Çetin and L.C.G. Rogers (2007) Modeling liquidity effects in discrete time Mathematical Finance 17 (1), 15-29.

M.G. Crandall, H. Ishii \& P. Lions (1992) User's guide to viscosity solutions of second order partial differential equations Bulletin of the American Mathematical Society 27 (1), 1-67.

J. Cvitanić \& I. Karatzas (1996) Hedging and portfolio optimization under transaction costs: A martingale approach Mathematical Finance 6 (2)m 133-165.

M.H.A. Davis \& A. R. Norman (1990) Portfolio Selection with Transaction Costs Mathematics of Operations Research 15 (4), 676-713.

A Semi-Lagrangian Approach For American Asian Options Under Jump Diffusion SIAM Journal on Scientific Computing 27 (1), 315-345.

G. Di Nunno \& B. Øksendal (2009) Optimal portfolio, partial information and Malliavin calculus Stochastics 81 (3-4), 303-322.

W.H. Fleming \& H.M. Soner (2006) Controlled Markov Processes and Viscosity Solutions. New York; Springer-Verlag.

W. Fung \& D. A. Hsieh (1997) Empirical Characteristics of Dynamic Trading Strategies: The Case of Hedge Funds, The Review of Financial Studies 10, 275-302.

W. Fung, D. A. Hsieh, N. Y. Naik, \& T. Ramadorai (2008) Hedge Funds: Performance, Risk, and Capital Formation The Journal of Finance LXIII (4), 1777-1803.

N. Gârleanu (2009) Portfolio choice and pricing in illiquid markets Journal of Economic Theory 144, 532-564.

W. N. Goetzmann, Jr J. E. Ingersoll \& S. A. Ross (2003) High-Water Marks and Hedge Fund Management Contracts The Journal of Finance 58, 1685-1717.

P. Guasoni \& J. Obłój (2013) The Incentives of Hedge Fund Fees and High-Water Marks Mathematical Finance 26 (2), 269-295.

O. Guéant \& J. Pu Option pricing and hedging with execution costs and market impact Mathematical Finance 27 (3), 803-831.

J.E. Hodder \& J.C. Jackwerth (2007) Incentive Contracts and Hedge Fund Management Journal of Financial and Quantitative Analysis 42, 811-826.

R. Jaeger (2003) All About Hedge Funds, The Easy Way to Get Started. McGraw-Hill. 
R. S. J. Koijen (2012) The Cross-Section of Managerial Ability, Incentives, and Risk Preferences Journal of Finance 69 (3), 1051-1098.

Y. Lan, N. Wang \& J. Yang (2013) The economics of hedge funds Journal of Financial Economics 110, 300-323.

F. A. Longstaff Optimal Portfolio Choice and the Valuation of Illiquid Securities (2001) The Review of Financial Studies 14 (2), 407-431.

O. Menoukeu Pamen, T. Meyer-Brandis, F. Proske \& H. Binti Salleh (2013) Malliavin calculus applied to optimal control of stochastic partial differential equations with jumps Stochastics 85 (3), 431-463.

R. C. Merton (1969) Lifetime Portfolio Selection under Uncertainty: The Continuous-Time Case The Review of Economics and Statistics 51 (2), 247-257.

B. Øksendal (2006) Stochastic Differential Equations, An Introduction With Applications, sixth edition. Springer.

B. Øksendal \& A. Sulem (2000) Applied Stochastic Control of Jump Diffusions. Springer.

H. E. Ramirez, P. V. Johnson, P. Duck \& S. Howell (2018) The optimal interaction between a hedge fund manager and investor Applied Mathematical Finance, 1-28.

M. Spiegelman \& R. Katz (2006) A Semi-Lagrangian Crank-Nicolson algorithm for the numerical solution of advection-diffusion problems. Geochemistry, Geophysics, Geosystems 7 (5).

A. Staninforth \& J. Côté (1991) Semi-Lagrangian Integration Schemes for Atmospheric Models - A Review Monthly Weather Review 119, 2206-2223.

N. Touzi (2013) Optimal Stochastic Control, Stochastic Target Problems, and backward SDE. New York. Springer.

N. Touzi \& B. Bouchard (2011) Weak Dynamic Programming Principle for Viscosity Solutions SIAM Journal on Control and Optimization 49, 948-962.

P. Wilmott (1995) The Mathematics of Financial Derivatives: A Student Introduction. Cambridge University Press. 